\title{
Entropy and correlations in a fluid of hard spherocylinders: The onset of nematic and smectic order
}

\author{
D. Costa $^{\dagger}$, F. Micali ${ }^{\dagger}$, F. Saija ${ }^{\ddagger}$, and P. V. Giaquinta ${ }^{\dagger *}$ \\ $\dagger$ Istituto Nazionale per la Fisica della Materia (INFM) and \\ Università degli Studi di Messina, Dipartimento di Fisica \\ Contrada Papardo, C.P. 50 - 98166 Messina, Italy \\ $\ddagger$ CNR - Istituto per i Processi Chimico-Fisici, sez. Messina \\ Via La Farina 237 - 98123 Messina, Italy
}

\begin{abstract}
Hard spherocylinders (cylinders of length $L$ and diameter $D$ capped at both ends with two hemispheres) provide a suitable model for investigating entropy-driven, mesophase formations in real colloidal fluids that are composed of rigid rodlike molecules. We performed extensive Monte Carlo simulations of this model fluid for elongations in the range $3 \leq L / D \leq 5$ and up to $L / D=20$, in order to investigate the relative importance of translational and orientational correlations allowing for the emergence of nematic or smectic order in the framework of the so-called residual multi-particle entropy (RMPE). The vanishing of this quantity, which includes the re-summed contributions of all spatial correlations involving more than two particles, signals the structural changes which take place, at increasing densities, in the isotropic fluid. We found that the ordering thresholds detected through the zero-RMPE condition systematically correlate
\end{abstract}

${ }^{*}$ Corresponding author; E-mail: Paolo.Giaquinta@unime.it 
with the corresponding phase-transition points, whatever the nature of the higher-density phase coexisting with the isotropic fluid.

PACS 64.60.-i, 64.70.Md, 61.20.Ja, 65.40.Gr

\section{Introduction}

A systematic approach to the study of the relation between configurational entropy, spatial correlations and local ordering in a fluid of elongated particles was recently outlined in [1], where the Authors used the theoretical framework that had been originally introduced by Giaquinta and Giunta in order to identify the "hidden" signature of freezing in the structural properties of a homogeneous and isotropic fluid of hard spheres [2]. Their approach was based on the well known multi-particle correlation expansion of the statistical entropy, first established for a finite system by H. S. Green [3] and later generalized to an infinite open system by R. E. Nettleton and M. S. Green [4]:

$$
s_{\mathrm{ex}}=\sum_{n=2}^{\infty} s_{n},
$$

where $s_{\text {ex }}$ is the excess entropy per particle in units of the Boltzmann constant and the " $n$-body entropies" $\left(s_{n}\right)$ are obtained from the integrated contribution of spatial correlations between $n$-tuples of particles. Note that the one-body term coincides with the entropy of the corresponding ideal gas. The

pair entropy of a homogeneous and isotropic fluid of nonspherical molecules can be written as [5]:

$$
s_{2}(\rho)=-\frac{1}{2} \frac{\rho}{\Omega^{2}} \int\left\{g\left(r, \omega^{2}\right) \ln \left[g\left(r, \omega^{2}\right)\right]-g\left(r, \omega^{2}\right)+1\right\} \mathrm{d} \mathbf{r} \mathrm{d} \omega^{2},
$$

where $g\left(r, \omega^{2}\right)$ is the pair distribution function (PDF) which depends on the relative separation $r$ between a pair of molecules and on the set of Euler angles $\omega^{2} \equiv\left[\omega_{1}, \omega_{2}\right]$ that specify the absolute orientations of the two particles in the laboratory reference frame. The quantity $\Omega$ represents the integral over the Euler angles of one molecule $(\Omega=4 \pi$ for linear molecules), while $\rho$ is the particle number density. 
The approach originally set up by Giaquinta and Giunta focused on the properties of the so-called residual multi-particle entropy (RMPE):

$$
\Delta s \equiv s_{\mathrm{ex}}-s_{2}
$$

The quantity $\Delta s$ includes, by definition, the re-summed contributions of all correlations involving more than two particles [2]. This quantity, despite its minor quantitative relevance in the overall entropy balance, is far from being irrelevant in that it conceals significant indications on the statistical thermodynamics of the fluid that are intimately related to the role played by high-order density correlations in the system. The noticeable feature exhibited by the RMPE, as compared to the pair entropy (a negative-definite quantity), is its non-monotonic behavior. In fact, $\Delta s(\rho)$ starts decreasing for increasing densities until the function attains a minimum beyond which it undergoes a sharp increase up to positive values. This behavior may come out, at first glance, somewhat unexpected in that one would probably surmise that, in analogy with the monotonic behavior of the two-body term $s_{2}$, the growth of higher-order density correlations that is induced by a further compression of the system should throughout contribute to a contraction of the configurational states that are accessible to the system for smaller and smaller volumes, as is actually the case from low to intermediate densities. Instead, one observes a "slowing down", for increasing pressures, in the reduction rate of the average number of states with respect to the non-interacting system in an equivalent thermodynamic condition: in fact, the positive cumulative contribution of multiparticle correlations to the entropic balance moderately damps the systematic drop that is caused by the dominant pair term. This effect is suggestive of the emergence of a new structural condition in the system. More specifically, it indicates that multiparticle correlations have started to build up some type of ordering, on a local scale, as a result of compelling entropic constraints. The associated signature is the vanishing of the RMPE: a posteriori, it is found to herald in a very sensitive way the occurrence - in a proximate range of densities and/or temperatures - of a phase transition of the fully disordered fluid into a more structured phase. By now, the reliability of such a one-phase ordering criterion has been extensively documented for a variety of model fluids $[6,7]$, also in two dimensions [8].

With specific reference to a fluid of hard spherocylinders, i.e., cylinders of length $L$ and diameter $D$ capped at both ends with two hemispheres, 
preliminary evidence was reported in [1] on the RMPE indication concerning the isotropic-nematic (I-N) transition. In the case of simple fluids composed of spherical particles a number of one-phase criteria have been introduced in the past with the aim of locating the transition from the liquid to the solid phase without resorting to the knowledge of the free energy of the two coexisting phases [9]. However, no such simple criteria are available for nematic fluids. In this respect, the zero-RMPE criterion stands as, perhaps, a unique exception in that it can be extended in a straightforward way to fluids composed of elongated particles to deal with the transition from the homogeneous and isotropic fluid to a liquid-crystalline phase.

Since the seminal work of Onsager [10], the model of hard spherocylinders has been diffusely investigated, notwithstanding its simplicity, in order to study excluded-volume effects in real liquid crystals. In fact, hard spherocylinders exhibit - depending on the value assumed by the aspect ratio $L / D$ - a surprising variety of mesophases $[11,12,13,14,15]$. In [1] constant-pressure Monte Carlo simulations were carried out for $L / D=5$ only, a case where the disordered fluid undergoes, under compression, a firstorder transition to a partially ordered nematic phase. In this paper we have extended the above calculations to lower values of the aspect ratio in the range $3 \leq L / D \leq 5$, thus encompassing a region where the homogeneous and isotropic fluid undergoes a transition to a fully ordered solid (S) phase as well as to the smectic-A (SmA) phase, a layered orientationally ordered phase characterized by a one-dimensional density modulation along the direction $\mathbf{n}$ of the molecular alignment [15]. We have carried out simulations also in the limit of very elongated particles, specifically for $L / D=20$, in order to analyze the behavior of the RMPE in a regime where the I-N transition point can be determined, asymptotically, in an exact way $[10,16,17]$. This is also the range of aspect ratios relevant for the modelling of monodisperse aqueous suspensions of rodlike particles like the tobacco mosaic virus, a plant virus which shows an isotropic-nematic transition as well as a smectic phase at higher concentrations [20].

The paper is organized as follows: The theoretical framework and the numerical simulation technique, as applied to the case of cylindrical molecules with head-tail symmetry, are described in Sects. 2 and 3, respectively. The results are presented in Sect. 4 while Sect. 5 is devoted to concluding remarks. 


\section{Theory}

In order to evaluate the RMPE from Eq. (3), one needs to calculate the excess and pair entropies of the fluid. The former quantity can be obtained by integrating the equation of state (EoS) along a thermodynamic path:

$$
s_{\mathrm{ex}}(\rho)=s_{\mathrm{ex}}(\bar{\rho})-\int_{\bar{\rho}}^{\rho}\left[\frac{\beta P}{\rho^{\prime}}-1\right] \frac{\mathrm{d} \rho^{\prime}}{\rho^{\prime}} .
$$

In Eq. (4) $P$ is the pressure, $\beta$ is the inverse temperature in units of the Boltzmann constant, and $\bar{\rho}$ is the density of a suitably chosen reference state. The EoS of the fluid was obtained through a Monte Carlo sampling of the system at different pressures, while the quantity $s_{\text {ex }}(\bar{\rho})$ was calculated using the Widom test-particle insertion method [21, 22] at low enough densities.

As for the pair entropy, this quantity was obtained, according to Eq. (2), after integration of the PDF which, in turn, was decoupled in the form [23]:

$$
g\left(r, \omega^{2}\right)=g(r) g\left(\omega^{2} \mid r\right),
$$

where $g(r)$ is the radial distribution function of the molecular centers of mass that is obtained after integrating the full PDF over the set of Euler angles, while $g\left(\omega^{2} \mid r\right)$ represents the conditional distribution function, normalized to $\Omega^{2}$, for the relative orientation of a pair of molecules whose centers of mass lie at a distance $r$. Using Eq. (5), the pair entropy was separated into the sum of a translational $\left(s_{2}^{\mathrm{tr}}\right)$ and an orientational part $\left(s_{2}^{\mathrm{or}}\right)$, after sorting out an additional excluded-volume contribution which arises in Eq. (2) from the integration over space regions where $g\left(r, \omega^{2}\right)=0$ :

$$
s_{2}=-B_{2} \rho+s_{2}^{\mathrm{tr}}+s_{2}^{\mathrm{or}} .
$$

In Eq. (6), $B_{2}$ is the second virial coefficient of hard spherocylinders:

$$
B_{2}=\frac{2}{3} \pi D^{3}+\pi D^{2} L+\frac{\pi}{4} D L^{2}
$$

The translational contribution is formally analogous to the pair entropy of a simple fluid with no rotational degrees of freedom:

$$
s_{2}^{\mathrm{tr}}=-\frac{1}{2} \rho \int[g(r) \ln g(r)-g(r)+1] \mathrm{d} \mathbf{r},
$$


where the integration is carried out over non-overlapping regions. As for the orientational term, one finds:

$$
s_{2}^{\mathrm{or}}=\rho \int g(r) S^{\mathrm{or}}(r) \mathrm{d} \mathbf{r}
$$

where

$$
S^{\text {or }}(r)=-\frac{1}{2 \Omega^{2}} \int g\left(\omega^{2} \mid r\right) \ln \left[g\left(\omega^{2} \mid r\right)\right] \mathrm{d} \omega^{2} .
$$

Even for uniaxial molecules, the computation of the full orientational correlation function $g\left(\omega^{2} \mid r\right)$ and its subsequent integration is a formidable task. In this specific case there are only four independent degrees of freedom which can be fixed by providing, e.g., the relative intermolecular distance $r$ and three angles [24]. Without loss of generality, we can hold the center of mass of, say, particle 1 fixed at the origin of the laboratory coordinate system and let its molecular axis $\mathbf{e}_{\mathbf{1}}$ lie along the $z$-axis. Then, the position and orientation of particle 2 can be unambiguously determined by specifying, for instance, two polar coordinates of its center of mass, i.e., the distance $r$ and the angle $\vartheta$ formed by the intermolecular axis with the $z$-axis, and the Euler angles $\theta$ and $\phi$ of the $\mathbf{e}_{2}$ axis (the "twist" angle, $\chi$, is redundant for uniaxial molecules). In order to simplify the whole scheme, we neglected the dependence of $g\left(\omega^{2} \mid r\right)$ on $\vartheta$ and $\phi$, thus focusing on the information associated with the angle representing the relative orientation of the two molecular axes, $\theta=\arccos \left(\mathbf{e}_{\mathbf{1}} \cdot \mathbf{e}_{\mathbf{2}}\right)$. Indeed, this is the critical parameter that should be monitored in order to assess the onset of either nematic or smectic order in the fluid. The contracted orientational distribution function $g(\theta \mid r)$ is normalized in such a way that:

$$
\frac{1}{2} \int_{0}^{\pi} g(\theta \mid r) \sin \theta \mathrm{d} \theta=1
$$

We do not expect that such a simplification may heavily bias the estimate of the weight associated with orientational correlations in the homogeneous and isotropic phase. Indeed, the quantity we are ultimately interested in is just an integral measure that can help to assess the relative balance between the positional and angular contributions that intervene in the configurational entropy. 


\section{Simulation}

We performed Monte Carlo (MC) simulations of hard spherocylinders at constant pressure. This choice is rather common for systems composed of non-spherical hard-core particles for which it may be difficult to calculate the equation of state through the contact value of the PDF, as is usually done in a constant-volume simulation. Furthermore, density fluctuations are more naturally accommodated in a constant-pressure simulation [22]. The number of particles, that were enclosed in a cubic box with periodic boundary conditions, typically ranged between $500(L / D=3.2,5)$ and 800 $(L / D=3)$, with an intermediate value of 672 particles for $L / D=4$. A larger sample composed of 1500 particles was used for more elongated particles with $L / D=20$.

A perfectly ordered, low-density lattice arrangement was the starting configuration. Then, the system was allowed to equilibrate, initially along a constant-volume MC path. A sequence of states was then generated at constant pressure, starting from an equilibrated isotropic configuration. We carried out rather extended simulations, as compared with those currently reported in the literature, especially in proximity of the transition threshold. In fact, after an equilibration period of typically $10^{5}$ to $10^{6} \mathrm{MC}$ cycles, simulation data were obtained by generating chains consisting of $5 \times 10^{5}$ to $5 \times 10^{6}$ $\mathrm{MC}$ cycles, depending on the pressure. A MC cycle consisted of an attempt of changing, sequentially, both the position and the orientation of each molecule, followed by an attempt of changing the volume of the sample. During such a procedure, each molecule was first displaced from its original position, by moving at random its center of mass within a cube. The molecule was then re-oriented within a cone centered about the molecular axis. The overall acceptance probability for the combined move was adjusted to about $50 \%$. The isotropic change of the simulation box was obtained by varying at random

the box side $L$. Finally, the maximum displacement allowed was set so as to achieve an average acceptance probability for volume changes of $\sim 50 \%$. For hard-core molecules, the usual Metropolis acceptance criterion reduces to a check of particle overlaps after each Monte Carlo move. In this regard, a simple and efficient algorithm can be used for spherocylinders [25, 26]. During the cumulation runs, we monitored the average density and built histograms for the translational and orientational distribution functions, respectively; equilibrium averages and standard deviations were computed by dividing 
such runs into 5 to 10 independent blocks. In the following, we shall refer to the volume packing fraction $\eta=\rho V_{\mathrm{hsc}}$, where $V_{\mathrm{hsc}}=(\pi / 4) D^{2} L+(\pi / 6) D^{3}$ is the volume occupied by one spherocylinder, and to the reduced pressure $P^{*}=\beta P V_{\mathrm{hsc}}$. We sampled the radial distribution function $g(r)$ at intervals of $0.1 D$, while the orientational distribution $g(\theta \mid r)$ was calculated with a grid spacing of $\Delta \theta=5^{\circ}$.

We investigated the phase diagram of the model up to the threshold of either the nematic or the smectic phase. The onset of liquid-crystalline order was monitored through the nematic order parameter:

$$
S=\frac{1}{N} \sum_{i=1}^{N} \frac{3 \cos ^{2} \psi_{i}-1}{2},
$$

where $\psi_{i}$ is the angle formed by the molecular axis of the $i$-th molecule $\mathbf{e}_{i}$ (the sum running over all particles in the system). The director $\mathbf{n}$ defines the alignment direction attained, on average, by the molecules. Since this direction is not known a priori, the order parameter is usually determined by diagonalizing the traceless, symmetric, second-rank tensor $\mathbf{Q}$ that is defined in terms of the molecular axes as:

$$
\mathbf{Q}=\frac{1}{N} \sum_{i=1}^{N} \frac{3 \mathbf{e}_{i} \mathbf{e}_{i}-\mathbf{I}}{2},
$$

where $\mathbf{I}$ is the unit tensor. The nematic order parameter is then obtained as the largest eigenvalue, and the director as the corresponding eigenvector [25]. The value attained by $S$ is close to zero in the isotropic phase and tends to one in a highly ordered phase. Across the ordering transition, both the density and the order parameter show a finite jump. However, such discontinuities, that are associated with a first-order phase transition, are in certain cases so small that a closer investigation of the correlation functions or a direct observation of the arrangement of the molecules in the sample may be necessary on the thermodynamic edge of both the nematic and smectic phase.

As already anticipated, we used Widom's insertion method [21, 22] to obtain independent numerical estimates of the excess entropy at low densities, $s_{\text {ex }}(\bar{\rho})$, to be used in Eq. (4). The Widom technique provides a simple scheme for calculating the excess chemical potential, $\mu_{\mathrm{ex}}$, of not too dense a liquid via the calculation of the average Boltzmann factor associated with the (never 
accepted) random insertion of an additional particle in an $N$-particle system. The excess entropy can then be obtained through the thermodynamic equation:

$$
s_{\mathrm{ex}}=-\beta \mu_{\mathrm{ex}}+\frac{\beta P}{\rho}-1
$$

Depending on the density and the elongation of the spherocylinders, 50 to 200 trial insertions per MC step were typically needed in order to obtain a reliable estimate of $\mu_{\mathrm{ex}}$. The use of this technique at small but finite densities avoids the otherwise necessary extrapolation to the ideal gas limit, as a reference state for the integration contemplated in Eq. (4). This latter procedure was adopted in [1], where some spurious fluctuations of the orientational distribution function as well as some difficulties associated with the simulation in the highly dilute regime were registered.

As sketched in Fig. 1, the values obtained for $s_{\text {ex }}$ using Widom's insertion method are quite stable in all the cases we have examined: the thermodynamic potential relaxes almost immediately, with small fluctuations about the average value. No significant drifts were observed during simulations as long as $10^{5} \mathrm{MC}$ steps. The simulation parameters and the results for the relevant properties of the fluid at low densities were collected for various elongations in Table 1.

\section{Results and discussion}

\subsection{Phase behavior for $3 \leq L / D \leq 5$}

In this subsection we shall focus on the phase behavior of the model in a range of parameters where, for increasing values of the aspect ratio, the disordered fluid undergoes a transition to either a solid, a smectic or a nematic phase. We investigated the model for shape anisotropies $L / D=3,3.2,4$, and 5 . For $L / D=3$, the fluid freezes into a crystalline phase. An I-SmA phase transition is observed over a range of larger aspect ratios bounded by an isotropic-smectic-solid (I-SmA-S) triple point $(L / D=3.1)$ and by an I-NSmA triple point $(L / D=3.7)$, respectively. For even larger anisotropies, the disordered fluid forms, under compression, a nematic phase [15].

The reliability of the present results for the EoS and for the nematic order parameter can be appraised through Fig. 2 where the above quantities 
were plotted as a function of the packing fraction for $L / D=3.2$ and 5 . The comparison with the simulation data reported by McGrother and coworkers [14] shows that a system with 500 particles is fairly adequate for evaluating the basic thermodynamic properties of the fluid. The agreement turned out to be equally good along the disordered branch also for $L / D=3$ and 4, up to the transition point.

We now turn to a discussion of the properties of the model for $L / D=5$, a case where the isotropic fluid undergoes a rather weak first-order transition to a thermodynamically stable nematic phase at about $45 \%$ of the closepacking density, a value corresponding to an absolute packing fraction of $0.4[14,15]$. No specific feature indicating the transition edge is apparent either in the equation of state or in the order parameter (see Fig. 2). A rather modest degree of positional order shows up in the spatial profile of the radial distribution function that was plotted in Fig. 3 for increasing pressures, all the way up to the transition threshold. Actually, for moderately small packing fractions, the local density around one generic spherocylinder is significantly reduced, with respect to the homogeneous value taken at large distances, for interparticle separations lower than $\sim 3 \frac{1}{2}$ molecular diameters, a distance which corresponds to the minimum contact separation between two orthogonal spherocylinders.

Retrospectively, this local "depletion" effect is more easily understood if one looks at the orientational distribution function (see Fig. 4): the almost flat profile of $g(\theta \mid r)$ for $r>3.5 D$, when plotted as a function of $\theta$, implies that orthogonal geometries in the relative spatial arrangement of two spherocylinders are equally probable as parallel configurations are. The steric hindrance produced, on average, by such orthogonal (or almost orthogonal) particles sensitively reduces the probability of finding other molecules at shorter distances from the central one. Upon further compressing the fluid, the value of $g(\theta \mid r)$ for aligned-particles geometries $(\theta=0, \pi)$ steadily increases while different geometries become, at the same time, rarer and rarer. This is apparent from the progressive and systematic reduction of $g(\theta \mid r)$, for short and intermediate molecular separations, over a wide range of angles centered about $\pi / 2$. Correspondingly, the radial distribution function becomes more and more structured at short distances as is distinctly shown in Fig. 3 by the building up of a first coordination shell formed by particles lying almost at close contact with the central one.

The excess entropy of the fluid was resolved in Fig. 5 in terms of the 
contributions associated with pair and more-than-two-particle correlations, respectively (see Eq. (3)). The behavior of the RMPE is similar to that exhibited by the same quantity in a variety of other model systems, composed of spherical particles, across an ordering transition such as the freezing of a fluid $[6,8]$ or the phase separation of a binary mixture [7]. In particular, this quantity vanishes for a value of the packing fraction $\left(\eta_{0}\right)$ which falls precisely on top of the currently estimated I-N phase transition point (see Table 2 where we summarized the predictions of the entropy-based criterion for the presently investigated values of the aspect ratio). We note that the current RMPE estimate sensitively improves on that formerly given in [1] where the values reported for the pair entropy turned out to be affected by a systematic computational error. However, no significant morphological change resulted, a posteriori, in both the pair entropy and in the RMPE. We also recall that the Authors had shown in [1] that the pair entropy changes with the size of the sample, for a given density, in a tiny but systematic way, the zero-RMPE density shifting towards moderately larger values. The RMPE inverts its trend (from decreasing to increasing) in the density region $(\eta \sim 0.36)$ corresponding to a more extended structuring of the fluid at short distances (see Fig. 3). To be specific, we refer to the growing of a local nucleus of (mostly) aligned particles that is signalled by the emergence of a secondary maximum in the radial distribution function at a relative distance twice as large as that corresponding to the first coordination shell.

Figure 5 also shows the pair entropy of the fluid resolved, according to Eq. (6), into the sum of a (spatially independent) excluded-volume term $\left(-B_{2} \rho\right)$ plus two more contributions arising from translational and orientational correlations, respectively. As expected, it is $s_{2}^{\text {or }}$ that drives the rather sharp increase of $\Delta s(\eta)$ just beyond the minimum. Vice versa, the contribution associated with positional correlations, $s_{2}^{\text {tr }}$, is very small all over the fluid range. The function which, upon integration (see Eq. (9)), yields the quantity $s_{2}^{\text {or }}$ is shown, for increasing densities, in the right part of Fig. 3. The value of the corresponding integral is negative and keeps close to zero at low and intermediate densities (see Fig. 5). For packing fractions larger than $\sim 0.3, s_{2}^{\text {or }}$ starts decreasing more and more rapidly and eventually overcomes (in absolute value) the excluded-volume term. As seen from Fig. 5, the fingerprint of the ordering of the fluid is manifest in the persistence of angular correlations up to intermediate and long distances.

Considerations similar to those developed above for $L / D=5$ apply also 
to the $L / D=3.2$ case, even if it is a smectic phase that is now formed by the isotropic fluid. However, the basic underlying mechanism which drives the first-order I-SmA transition is analogous to that exploited in the nematic case. Obviously, the denser isotropic fluid looks more structured than for $L / D=5$ (see Fig. 6). Furthermore, at the transition point angular correlations show a very slow spatial decay. We also note that in this case the lower molecular asymmetry modifies the scale of interparticle distances in the average density profile. Thus, the effect associated with the structuring of the second coordination shell, which we already commented upon for $L / D=5$, is less resolved since the corresponding distance falls very close to the position of the lone weak maximum, located at a distance $r / D=1+\frac{1}{2}(L / D)=2.6$, that is present in the radial distribution function at low densities. A shoulder, rather than a peak, emerges at $r / D \simeq 2.4$, again in a density range corresponding to the position of the minimum $(\eta \simeq 0.47)$ attained by the RMPE (see Fig. 7).

In general, the ordering thresholds detected through the vanishing of the RMPE correlate very well with the corresponding transition point (see Table 2), whatever the nature of the higher-density phase coexisting with the isotropic fluid. The evidence discussed so far justifies the claim that the RMPE of spherocylinders monitors the leading microscopic process which drives the phase transition in all such cases, i.e., the aligning of spherocylinders along a common direction. As such, the vanishing of this quantity presumably portends the density threshold beyond which the nematic ordering may possibly emerge in the system, regardless of other concurrent structural phenomena like those which may ultimately lead to the formation of a more ordered macroscopic state, be it smectic or crystalline, instead of a pure nematic phase. An indication consistent with this thesis that is, perhaps, not accidental (yet to be considered with due caution because of the numerical uncertainty arising from the computational error which affects, at least, the third significant figure) is offered by the apparently greater accuracy of the predictions given by the RMPE-based criterion for $L / D=4$ and 5 (see Table 2), viz., in those cases where the stable coexisting phase is a truly nematic fluid. In the other two cases investigated, i.e., when either a solid or a smectic phase is formed, the criterion just overshoots the target, locating the internal threshold for the ordering of the fluid at a slightly higher density. 


\subsection{The Onsager regime}

As the aspect ratio increases, the I-N transition point moves to lower and lower densities. In the Onsager limit of infinitely long rods $(L / D \rightarrow \infty)$, a fluid of hard spherocylinders undergoes a first-order phase transition to a nematic phase for $B_{2} \rho=3.29[10,16,17]$. However, the phase behavior characteristic of the Onsager regime qualitatively sets in for shape anisotropies even less than $L / D=20$, even if for such values of the aspect ratio the basic assumption made by Onsager that all virial coefficients of order higher than two can be neglected is not yet satisfied [15]. In fact, the contribution of the third virial term to the free energy, evaluated for $L / D=20$ at the transition density, turns out to be still comparable to the contribution of the second virial term $[18,19]$.

We carried out simulations for $L / D=20$. In passing, we recall that this value mimics the typical size of the tobacco mosaic virus, whose particles have a practically rigid rod shape of $15-18 \mathrm{~nm}$ diameter and $300 \mathrm{~nm}$ length [20]. For such large values of the aspect ratio, the simulation of a fluid of hard spherocylinders dictates more severe constraints on the size of the simulation box which should be large enough to accommodate at least two rod lengths, so as to avoid multiple overlaps between a molecule and the periodic images of another particle [15]. The number of particles chosen $(N=1500)$ is sufficient to ensure that the condition mentioned above is fulfilled in the (relatively low) density range where the disordered phase is thermodynamically stable. The reduced pressure and the nematic order parameter are shown in Fig. 8. The isotropic liquid phase is mechanically stable up to a reduced pressure $P^{*}=0.92$ : in this regime the system shows, for simulations as long as $2.5 \times 10^{6} \mathrm{MC}$ cycles, no significant fluctuations about the average values of the packing fraction $(\langle\eta\rangle=0.145)$ and of the nematic order parameter $(\langle S\rangle=0.058)$, respectively. Upon further compressing the system up to $P^{*}=0.93$, we observed, after about $1.5 \times 10^{6} \mathrm{MC}$ cycles, a spontaneous and rapid transformation into a nematic phase which, at equilibrium, settled down at a packing fraction $\langle\eta\rangle=0.182$ and was characterized by an order parameter $\langle S\rangle=0.862$. The resulting "boundaries" are slightly shifted toward higher densities if compared with the direct estimates reported in [15], i.e., $\eta_{\text {iso }}=0.139$ or 0.137 , and $\eta_{\text {nem }}=0.171$ or 0.172 , according to whether the Gibbs-Duhem integration or a Gibbs-ensemble technique was used. Exploratory runs performed by decompressing the system from 
the ordered phase distinctly show an hysteresis, the fluid remaining almost nematic for pressures as low as $P^{*} \sim 0.75$. We calculated the pressure also using the "decoupling approximation" between the translational and orientational degrees of freedom originally introduced by Parsons [27]. In this approximation a system of rods is mapped onto a system of spheres interacting with an effective orientation-dependent potential. As already observed by McGrother and co-workers for the shorter elongation regime $3.2 \leq L / D \leq 5$ that was analyzed in [14], the resulting EoS turns out to be fairly accurate in the isotropic phase up to the transition point, and definitely better than the prediction based on the scaled-particle-theory approach exploited by Boublik [28]. However, it should be noted that the latter approach is based on a one-parameter approximation for the third and fourth virial coefficients which cannot be expected to yield accurate results for large elongations [29]. A better agreement with the simulation data is obtained with a closely related description that was proposed by Nezbeda [29, 30]: this approximation is based on an empirical fit of the virial coefficients, especially tailored on the available results for hard spherocylinders. All the above approximations for the EoS were reported, for a comparison, in Fig. 8: note that the simulation data are closely bracketed by the Parsons and Nezbeda approximations, respectively.

Radial correlations are shown in Fig. 9 together with the function whose space integral yields $s_{2}^{\text {or }}$. All over the liquid density range, the radial distribution function is practically structureless apart from the correlation hole at short distances (see Fig. 9). As a result, the translational contribution to the pair entropy keeps close to zero all over the explored density range (see Fig. 10). The same is also true for the orientational contribution for packing fractions less than about 0.15. It thus follows that the pair entropy of the isotropic fluid is substantially equal to the excluded-volume term $-B_{2} \rho$, as is actually assumed in the original Onsager theory. At variance with the positional term, the angular contribution is responsible, together with the excluded-volume term, of the vanishing of the RMPE. Just beyond the transition point, $s_{2}^{\text {or }}$ blows up and definitely overcomes the excluded-volume term (see Fig. 10). The resulting RMPE shows the typical behavior already observed for smaller aspect ratios. Also in this case, the zero-RMPE threshold is in very good agreement with the I-N transition point estimates given in [15] (see Table 2). We also reported in Fig. 10 the Parsons and Nezbeda analytical estimates for the excess entropy. As already pointed out for the 
EoS, both approximations turn out to predict fairly well, almost with the same degree of accuracy, also the excess entropy of the fluid.

As seen from Figs. 5, 7 and 10, the overall importance of the cumulative contribution of higher-order density correlations to the excess entropy decreases with increasing shape anisotropies. This is clearly a consequence of the progressive confinement of the isotropic phase to lower and lower densities. In this regard, we just observe that the value attained by the RMPE at its minimum drops from $\sim 45 \%$ of the excess entropy for $L / D=3.2$ to $\sim 18 \%$ for $L / D=20$. This behavior is consistent with the numerical findings on the virial expansion cited above and with the asymptotic trend predicted by Onsager as $L / D \rightarrow \infty$. Note, however, that at low densities the ratio $\Delta s / s_{\text {ex }}$ moderately increases with $L / D$ for a given packing fraction. Actually, longer and thinner particles give rise to more extended multiparticle correlations which trigger the ordering of the fluid at lower and lower densities. Beyond the zero of the RMPE, where the nematic phase is thermodynamically stable, the present data monitor de facto a system in a metastable, macroscopically isotropic condition whose proper description lies beyond the standard Onsager theory.

\section{Concluding remarks}

In this paper we have analyzed the contributions to the "pair entropy" $s_{2}$ of hard spherocylinders of length $L$ and diameter $D$ that are associated with excluded-volume, translational and angular correlations, with particular emphasis on the thermodynamic behavior exhibited by the above quantities on approaching the phase transition from the low-density fully disordered phase to a partially ordered phase at higher densities. An elucidating synthesis of the results obtained for $s_{2}$ through an intensive Monte Carlo sampling of the model is offered by Fig. 11 where we plotted the three contributions cited above, each of them being calculated in the state corresponding to the vanishing of the residual multi-particle entropy (RMPE) for an assigned value of $L / D$. The RMPE is a quantity that gauges the cumulative weight of spatial correlations involving more than two particles in the configurational entropy of the fluid. Figure 11 neatly indicates the relative importance of the three contributions in the overall entropic balance: for very small asymmetries, the translational term, $s_{2}^{\text {tr }}$, prevails (in absolute value) while being compa- 
rable with the excluded-volume contribution, $-B_{2} \rho$ (note that the data for $L / D=0$ do obviously refer to the hard-sphere case [2]). For increasing values of the aspect ratio the angular term, $s_{2}^{\text {or }}$, acquires more and more importance: in fact, an interpolation of the available data shows that the three contributions reach the same magnitude for $L / D \simeq 0.4$. Actually, it is in this range of values (more precisely, for $L / D \leq 0.35 \pm 0.05$ [15]) that the "rotator phase" (i.e., the plastic crystal) disappears in the phase diagram of hard spherocylinders, and is replaced at high densities by an orientationally ordered, crystal phase. As seen from Fig. 11, in the range $0.4 \lesssim L / D \lesssim 5$ the ordering of the fluid is mainly controlled by angular correlations. In fact, the translational contribution rapidly tends to smaller and smaller values and practically vanishes for $L / D>5$. Also $s_{2}^{\text {or }}$ asymptotically tends to zero in this range of increasing asymmetries but, as seen from Fig. 11, the decay of this quantity is much slower than that shown by its translational counterpart. Correspondingly, the excluded-volume term becomes the dominant contribution to the configurational entropy of the fluid. Note that its value for $L / D=20(-3.45)$ is already very close to the Onsager limit $(-3.29)$.

As far as the quality of the "predictions" offered by the zero-RMPE criterion are concerned, the agreement with the properly defined thermodynamic thresholds is impressive for all the asymmetries that have been investigated. Indeed, the vanishing of the RMPE monitors, in a very sensitive and reliable way, the ordering of the homogeneous and isotropic fluid into a more ordered phase be it nematic, smectic or solid. However, we remark that such a close quantitative correspondence should not be necessarily and systematically expected a priori. In fact, the zero of $\Delta s$ needs not coincide with the thermodynamic edge of the disordered phase that is properly located through the comparison of the free energies of the two coexisting phases. Actually, the RMPE conveys a type of information that is intermediate between the fully macroscopic level and the underlying microscopic description of the system. The interpretation of such a simply accessible information (and, more specifically, of its change of sign) as an intrinsic signature of the incipient ordering of the fluid appears to be quite firmly established on the basis of an ample and coherent evidence that has, by now, emerged in a variety of continuum and lattice model fluids $[6,7,8]$. The present analysis of the "fine structure" of the configurational entropy of hard spherocylinders further corroborates the sensitivity of the RMPE to local ordering phenomena which may even turn out into a rather weak first-order phase transition. We believe that this 
one-phase entropic criterion can be conveniently generalized to account for more than one phase transition, leading the system to increasing levels of self-organization. An attempt in this direction has been recently done by Cuetos and coworkers whose results seem to indicate the existence of a jump in the translational part of the pair entropy as well as in its rate of change across the nematic-smectic transition [32]. However, this evidence was not considered conclusive because of the limited size of the simulation box. We are also currently investigating this point with promising preliminary results.

\section{Acknowledgments}

We would like to thank Professor Bruno Martinez-Haya for useful discussions and for pointing a numerical inconsistency out in a previously published article. D. C. gratefully acknowledges the hospitality and advice of Professor M. P. Allen during a visit to the University of Bristol.

\section{References}

[1] D. Costa, F. Saija, and P. V. Giaquinta, Chem. Phys. Lett. 283, 86 (1998); Erratum: 299, 252 (1999).

[2] P. V. Giaquinta and G. Giunta, Physica A 187, 145 (1992).

[3] H. S. Green, The Molecular Theory of Fluids (North-Holland, Amsterdam, 1952).

[4] R. E. Nettleton and M. S. Green, J. Chem. Phys. 29, 1365 (1958).

[5] T. Lazaridis and M. E. Paulaitis, J. Phys. Chem. 96, 3847 (1992); 98, 635 (1994).

[6] F. Saija, S. Prestipino and P. V. Giaquinta, J. Chem. Phys. 115, 7586 (2001), and references contained therein.

[7] F. Saija and P. V. Giaquinta, J. Phys. Chem. B 106, 2035 (2002).

[8] F. Saija, S. Prestipino and P. V. Giaquinta, J. Chem. Phys. 113, 2806 (2000). 
[9] P. A. Monson and D. A. Kofke, Adv. Chem. Phys. 115, 113 (2000).

[10] L. Onsager, Ann. N.Y. Acad. Sci. 51, 627 (1949).

[11] D. Frenkel, J. Phys. Chem. 92, 3280 (1988).

[12] D. Frenkel, H. N. W. Lekkerkerker, and A. Stroobants, Nature 332, 822 (1988).

[13] J. A. C. Veerman and D. Frenkel, Phys. Rev. A 41, 3237 (1989).

[14] S. C. McGrother, D. C. Williamson and G. Jackson, J. Chem. Phys. 104, 6755 (1996).

[15] P. Bolhuis and D. Frenkel, J. Chem. Phys. 106, 666 (1997).

[16] H. N. W. Lekkerkerker, Ph. Coulon, R. van der Haegen, and R. Deblieck, J. Chem. Phys. 80, 3427 (1984).

[17] D. C. Williamson and G. Jackson, Molec. Phys. 83, 603 (1994).

[18] J. P. Straley, Mol. Cryst. Liq. Cryst. 22, 333 (1973); 24, 7 (1973).

[19] D. Frenkel, J. Phys. Chem. 91, 4912 (1987); 92, 5314 (E) (1988)

[20] For a review see S. Fraden in Proceed. of the NATO-ASI Observation, Prediction and Simulation of Phase Transitions in Complex Fluids, M. Baus, L. F. Rull and J. P. Ryckaert eds. (Kluwer, Dordrecht, 1994); N. Urakami, M. Imai, Y. Sano, and M. Takasu, J. Chem. Phys. 111, 2322 (1999); H. Graf and H. Löwen, Phys. Rev. E 59, 1932 (1999).

[21] B. Widom, J. Chem. Phys. 39, 2808 (1963).

[22] D. Frenkel and B. Smit, Understanding Molecular Simulation (Academic Press, London, 1996).

[23] T. Lazaridis and M. Karplus, J. Chem. Phys. 105, 4294 (1996).

[24] See, for example: A. K. Soper, C. Andreani, and M. Nardone, Phys. Rev. E 47, 2598 (1993). 
[25] M. P. Allen, G. T. Evans, D. Frenkel, and B. M. Mulder, Adv. Chem. Phys. 86, 1 (1993).

[26] C. Vega and S. Lago, Comp. Chem. 18, 55 (1994).

[27] J. D. Parsons, Phys. Rev. A 19, 1225 (1979).

[28] T. Boublik, Mol. Phys. 42, 209 (1981).

[29] For a review see: T. Boublik and I. Nezbeda, Collect. Czech. Chem. Comm. 51, 2301 (1986).

[30] I. Nezbeda, Chem. Phys. Lett. 41, 55 (1976).

[31] J. S. van Duijneveldt and M. P. Allen, Molec. Phys. 92, 855 (1997).

[32] A. Cuetos, B. Martinez-Haya, L. F. Rull, and S. Lago, J. Chem. Phys. 117, 2934 (2002). 


\section{Tables}

Table 1: Thermodynamic properties of the fluid in the low-density states $\bar{\rho}$ used for the integration of the equation of state in Eq. (4) of the text. Column VIII refers to the number of trial insertions per MC cycle; the percentage ratio of accepted trial insertions is reported in column IX. Standard deviations in the last digit(s) are given in parenthesis.

\begin{tabular}{lllllllcl}
$L / D$ & $P^{*}$ & $\langle\eta\rangle$ & $\left\langle\beta \mu_{\mathrm{ex}}\right\rangle$ & $\left\langle s_{\mathrm{ex}}\right\rangle$ & $\left\langle s_{2}\right\rangle$ & MC cycles & Trials & Ratio (\%) \\
\hline \multirow{3}{3}{3} & 0.30 & $0.131(1)$ & $2.33(1)$ & $-1.039(13)$ & $-0.959(2)$ & $5 \times 10^{5}$ & 100 & 9.7 \\
3.2 & 0.30 & $0.130(1)$ & $2.37(1)$ & $-1.054(17)$ & $-0.971(3)$ & $9 \times 10^{5}$ & $50 \div 200$ & 9.4 \\
4 & 0.30 & $0.125(1)$ & $2.55(1)$ & $-1.149(6)$ & $-0.996(1)$ & $5 \times 10^{5}$ & $80 \div 200$ & 7.8 \\
5 & 0.181 & $0.089(1)$ & $1.90(1)$ & $-0.878(6)$ & $-0.834(1)$ & $6 \times 10^{5}$ & 20 & 14.9 \\
& 0.379 & $0.136(1)$ & $3.25(1)$ & $-1.457(24)$ & $-1.237(4)$ & $5 \times 10^{5}$ & 200 & 3.8 \\
20 & 0.10 & $0.044(1)$ & $2.39(1)$ & $-1.137(13)$ & $-1.066(1)$ & $5 \times 10^{5}$ & 50 & 9.2 \\
& 0.20 & $0.066(1)$ & $3.79(1)$ & $-1.785(26)$ & $-1.588(5)$ & $10 \times 10^{5}$ & 200 & 2.2
\end{tabular}


Table 2: The packing fractions corresponding to the vanishing of the residual multiparticle entropy are compared, for different elongations, with the thermodynamic thresholds of the phase transitions undergone by the isotropic fluid, according to the available simulation data. Note that the value ascribed to Ref. [15] for $L / D=4$ was estimated through a linear interpolation of the data for the I-N transition densities reported in Ref. [15] for $L / D=3.8$ and $L / D=4.2$, respectively. A slightly lower estimate for the I-N transition density at $L / D=4, \eta=0.459$, is reported in Ref. [31].

Phase transition point

$L / D \quad$ Phase transition $\quad \eta_{0} \quad$ Ref. [14] Ref. [15]

\begin{tabular}{lcccc}
\hline & & & & \\
3.2 & I-S & 0.521 & 0.490 & 0.512 \\
4 & I-SmA & 0.510 & 0.513 & 0.503 \\
5 & I-N & 0.463 & 0.472 & 0.466 \\
20 & I-N & 0.399 & 0.407 & 0.398 \\
& I-N & 0.148 & - & 0.139
\end{tabular}




\section{Figures}

Figure 1: Typical evolution of $s_{\mathrm{ex}}(\bar{\rho})$ (see Table 1) during a MC run for different elongations. The data were obtained using the Widom test method. Circles: $L / D=3.2$ at $P^{*}=0.30$; diamonds: $L / D=5$ at $P^{*}=0.379$; squares: $L / D=20$ at $P^{*}=0.20$.

Figure 2: Equation of state (top) and nematic order parameter (bottom) for $L / D=3.2$ (left panels) and 5 (right panels). Solid circles: this work; triangles: MC simulations by McGrother et al. [14]. The error bars are systematically smaller than the size of the markers.

Figure 3: Radial distribution function (left) and integrand (right) for $s_{2}^{\text {or }}$ in Eq. (9) at several densities for $L / D=5$. Open triangles: $P^{*}=0.18$, $\langle\eta\rangle=0.089$; solid circles: $P^{*}=1.10,\langle\eta\rangle=0.223$; squares: $P^{*}=2.53$, $\langle\eta\rangle=0.310$; solid triangles: $P^{*}=4.20,\langle\eta\rangle=0.372$; diamonds: $P^{*}=4.95$, $\langle\eta\rangle=0.397$. 
Figure 4: Orientational distribution functions $g(\theta \mid r)$ at $P^{*}=1.10$ (left) and 4.95 (right), plotted, from top to bottom, for several distances $(r / D=1.25$, $1.95,2.55,3.65,6.05)$.

Figure 5: Left panel: residual multiparticle entropy (circles) resolved into the excess (squares) and pair (solid diamonds) contributions for $L / D=5$; the values of the excess entropy at lower densities were also computed using the Widom test method (pluses). Right panel: pair entropy (solid diamonds) resolved into the translational (triangles), orientational (squares), and excluded-volume (circles) contributions, according to Eq. (6). Lines are smooth interpolations of the simulation data.

Figure 6: Radial distribution function (left) and integrand (right) for $s_{2}^{\text {or }}$ in Eq. (9) at several densities for $L / D=3.2$. Open triangles: $P^{*}=0.5,\langle\eta\rangle=$ 0.170; solid circles: $P^{*}=2.0,\langle\eta\rangle=0.305$; squares: $P^{*}=5.0,\langle\eta\rangle=0.411$; solid triangles: $P^{*}=8.0,\langle\eta\rangle=0.474$; diamonds: $P^{*}=9.8,\langle\eta\rangle=0.507$; pluses (right panel): $P^{*}=9.9,\langle\eta\rangle=0.511$.

Figure 7: Left panel: residual multiparticle entropy (circles) resolved into the excess (squares) and pair (solid diamonds) contributions for $L / D=3.2$; the excess entropy at the lowest packing fraction was computed using the Widom test method. Right panel: pair entropy (solid diamonds) resolved into the translational (triangles), orientational (squares), and excluded-volume (circles) contributions, according to Eq. (6). Lines are smooth interpolations of the simulation data.

Figure 8: Left panel: equation of state for $L / D=20$ (solid circles); the Parsons (dotted line, [27]), Boublik (solid line, [28]), and Nezbeda (dashed line, [30]) approximations for the equation of state are also shown. Right panel: nematic order parameter plotted as a function of $\langle\eta\rangle$. Triangles refer in both panels to states obtained upon decompressing the nematic fluid. The error bars are systematically smaller than the size of the markers. 
Figure 9: Radial distribution function (left) and integrand (right) for $s_{2}^{\text {or }}$ in Eq. (9) at several densities for $L / D=20$. Circles: $P^{*}=0.10,\langle\eta\rangle=0.044$; squares: $P^{*}=0.50,\langle\eta\rangle=0.107$; triangles: $P^{*}=0.85,\langle\eta\rangle=0.140$; solid diamonds: $P^{*}=0.93,\langle\eta\rangle=0.182$; pluses (right panel): $P^{*}=0.70,\langle\eta\rangle=$ 0.127 .

Figure 10: Left panel: residual multiparticle entropy (circles) resolved into the excess (squares) and pair (solid diamonds) contributions for $L / D=20$; the excess entropy at lower densities was also computed using the Widom test method (pluses). The Parsons (dotted line, [27]) and Nezbeda (dashed line, [30]) approximations for the excess entropy are also shown. Right panel: pair entropy (solid diamonds) resolved into the translational (triangles), orientational (squares), and excluded-volume (circles) contributions, according to Eq. (6). Solid lines are smooth interpolations of the simulation data.

Figure 11: Translational (circles), orientational (squares), and excludedvolume (solid diamonds) contributions to the pair entropy of the fluid, calculated in the states of vanishing residual multiparticle entropy, for different shape anisotropies. Data for $L=0$ (hard spheres) were taken from Ref. [2]; data for $L=1$ were obtained from the equation of state reported in Ref. [13]. 


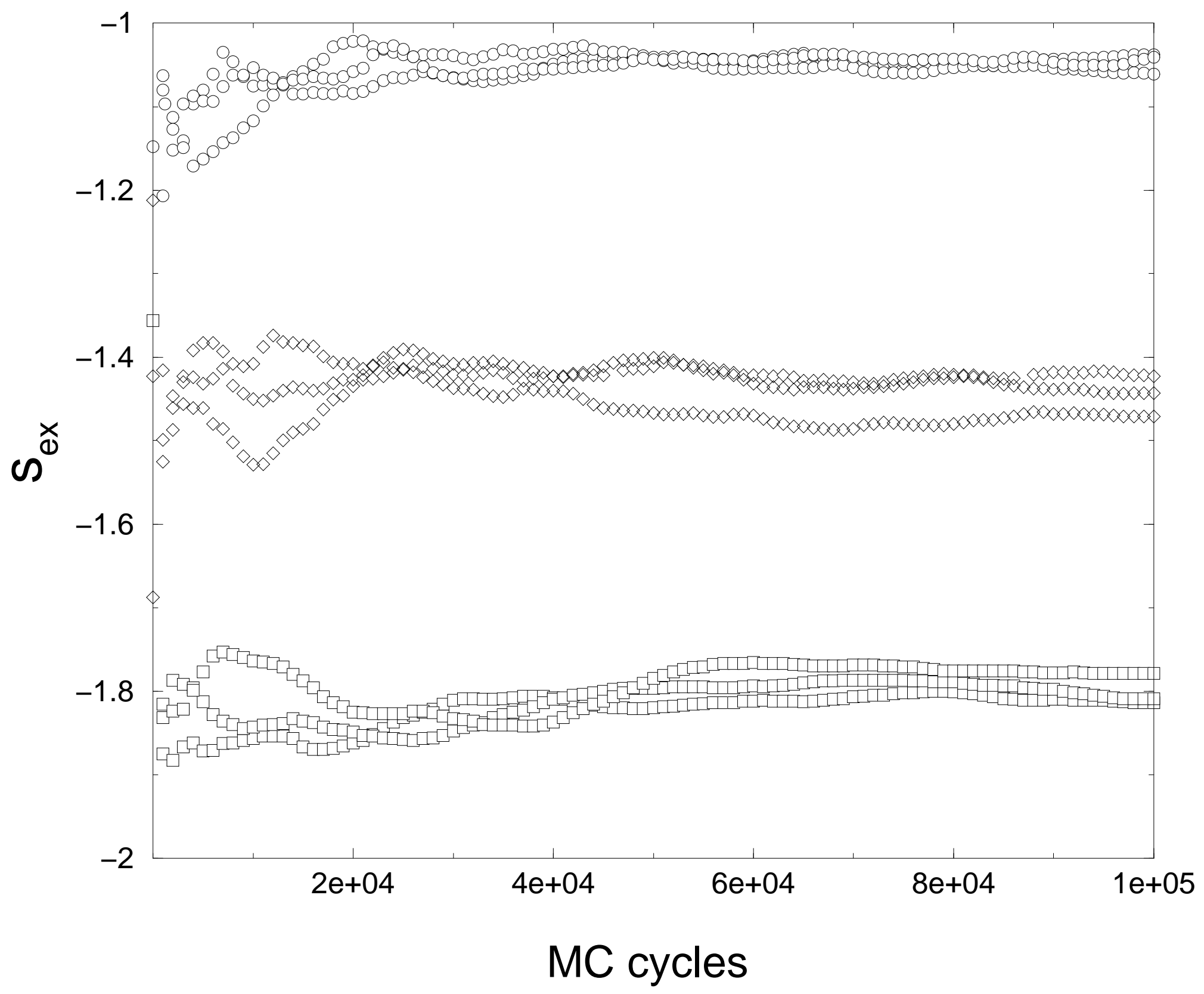




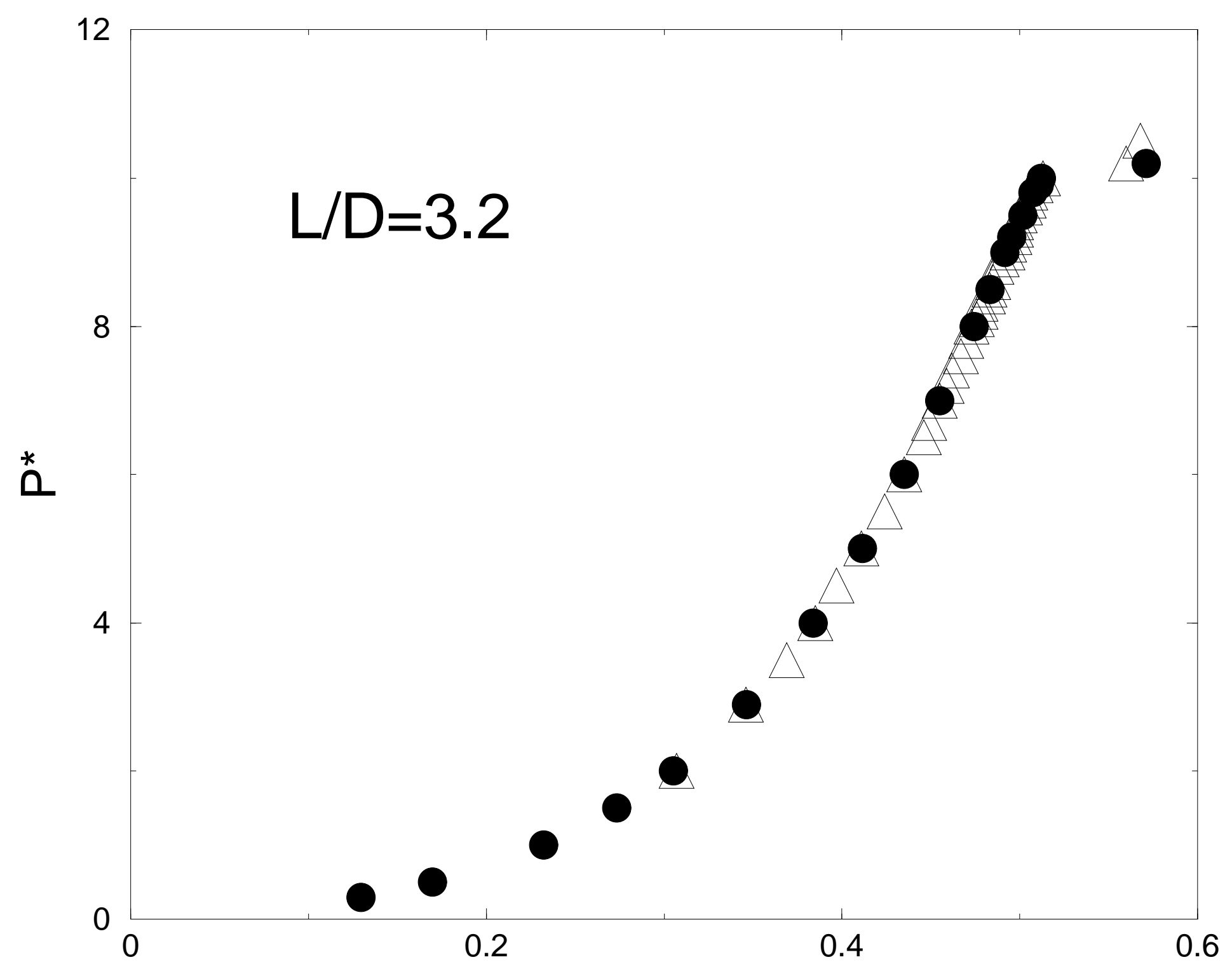




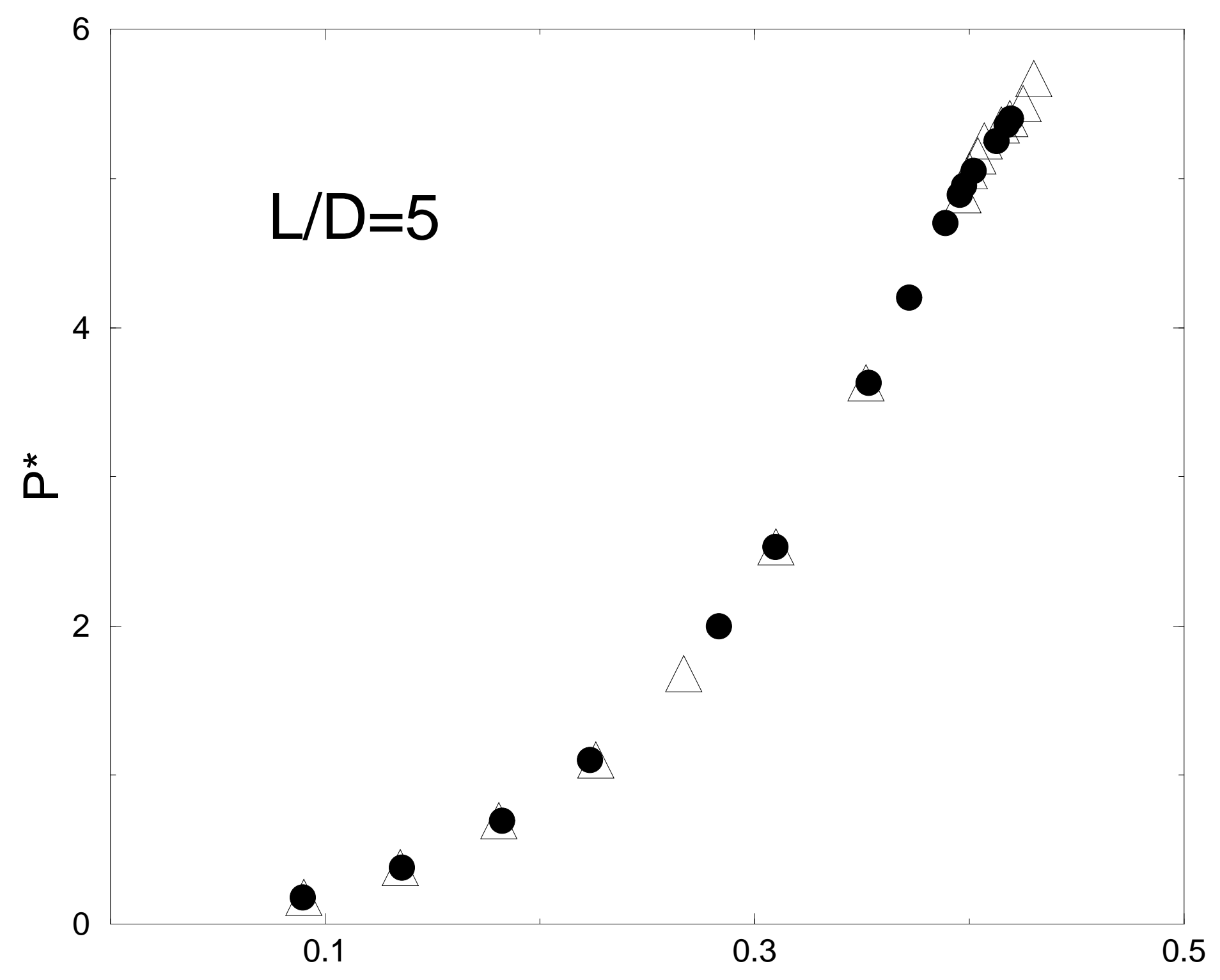




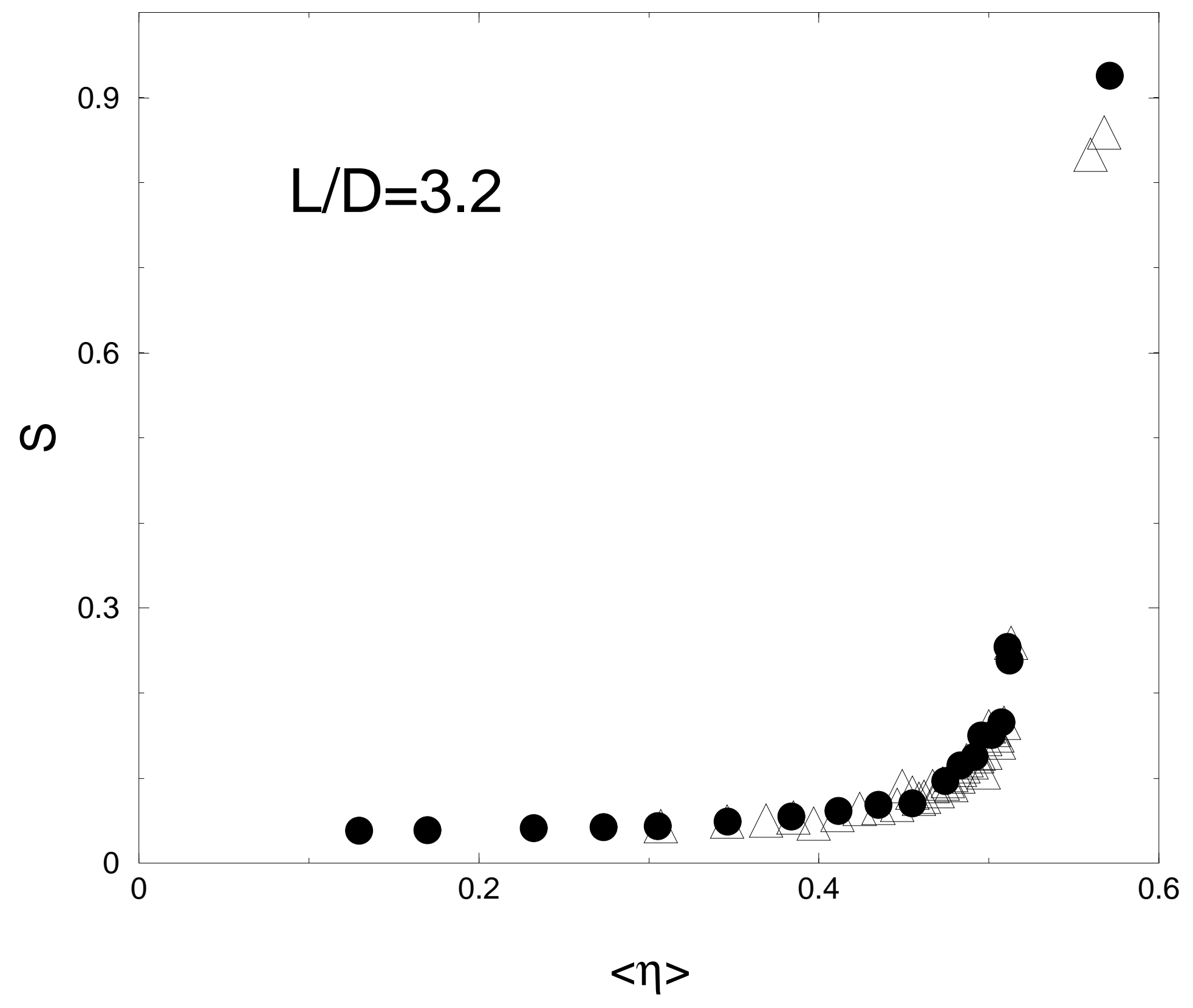




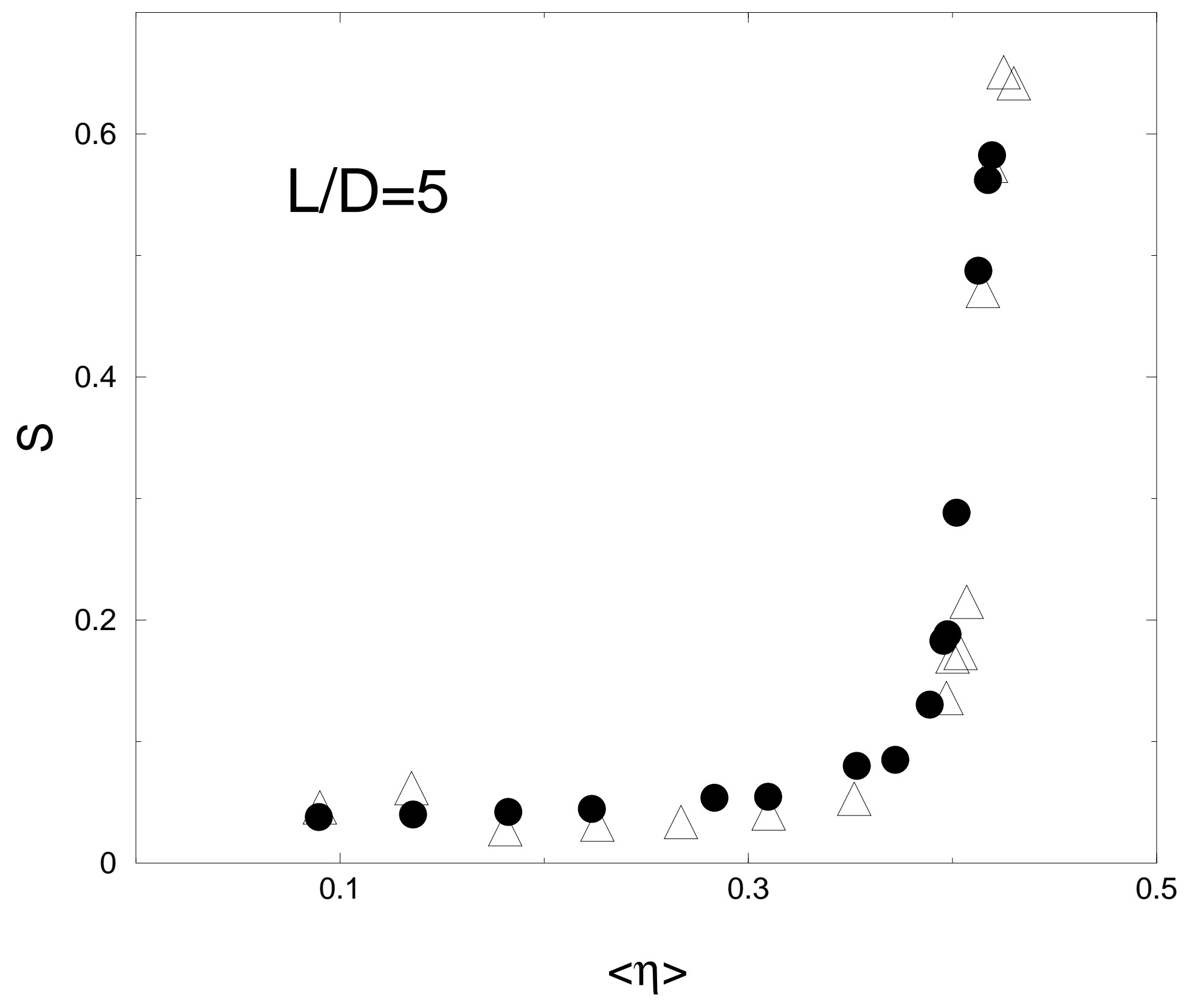




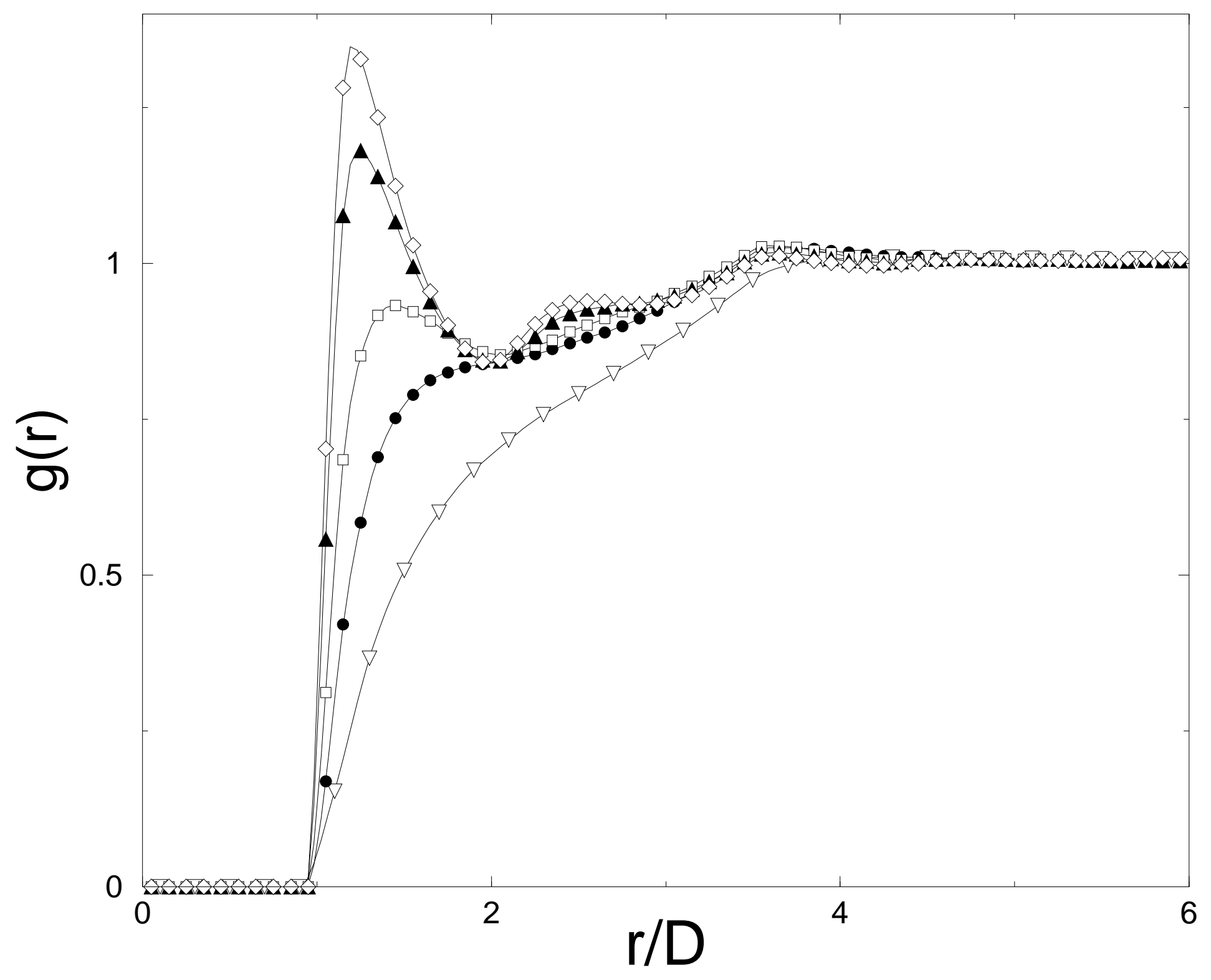




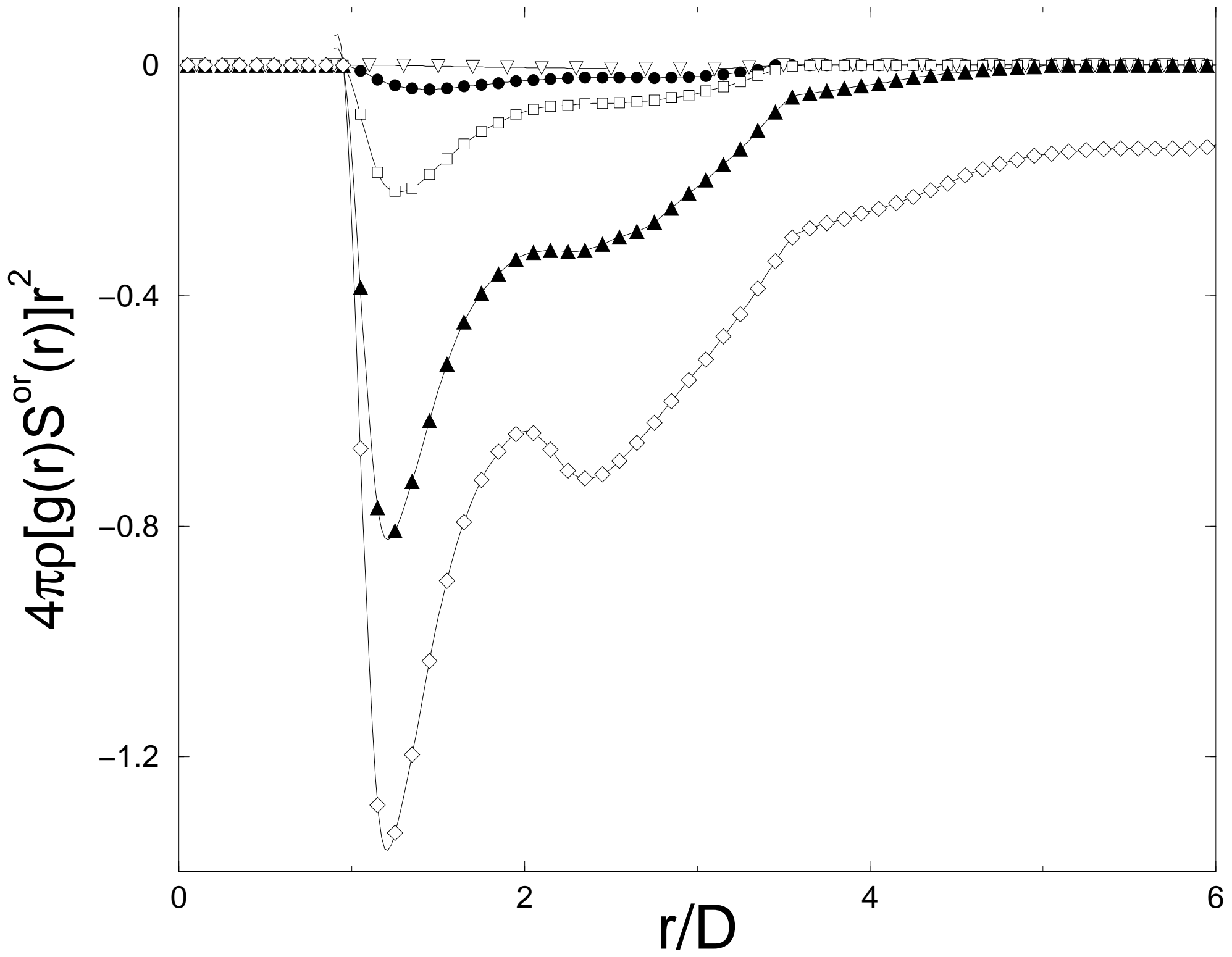




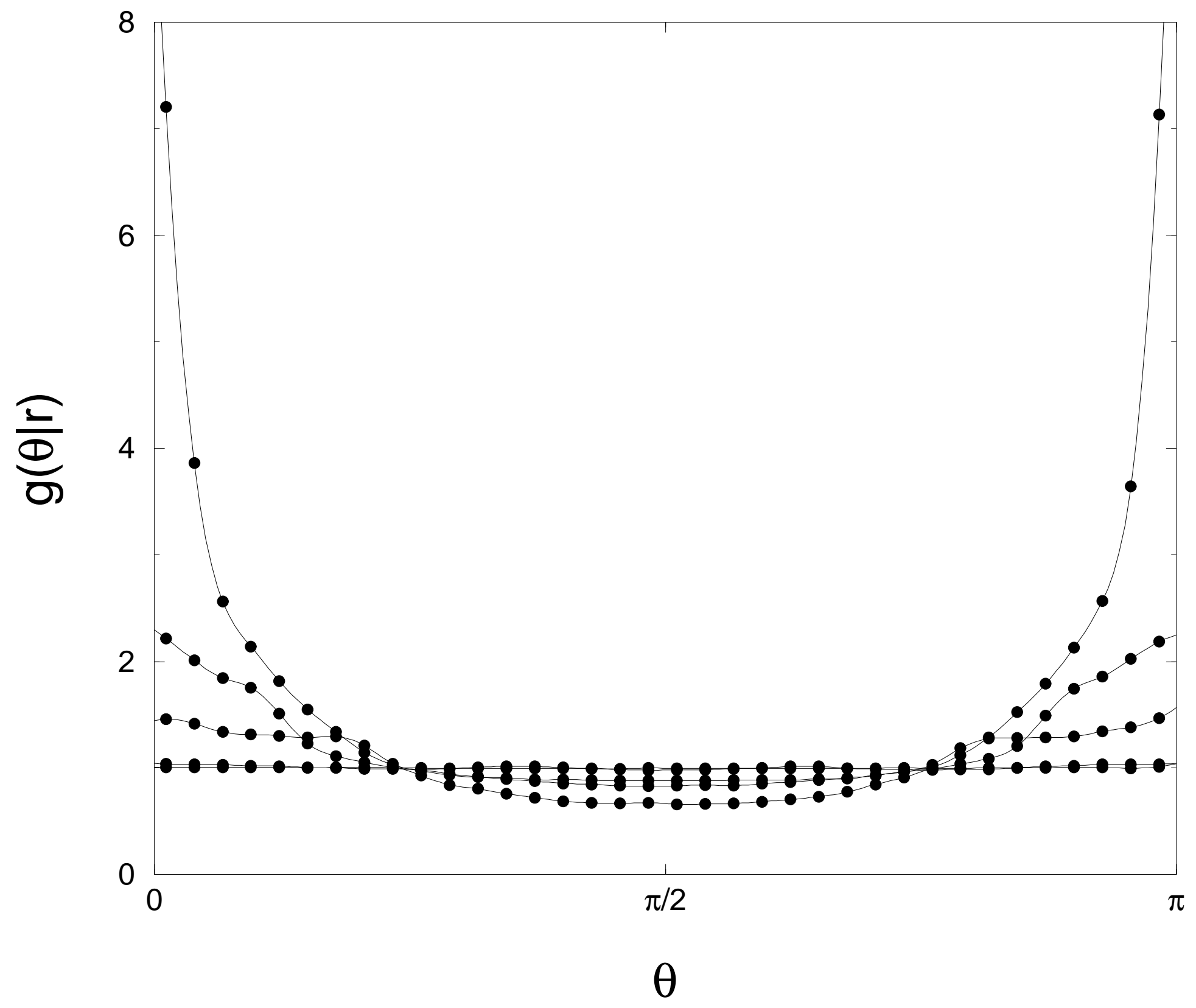




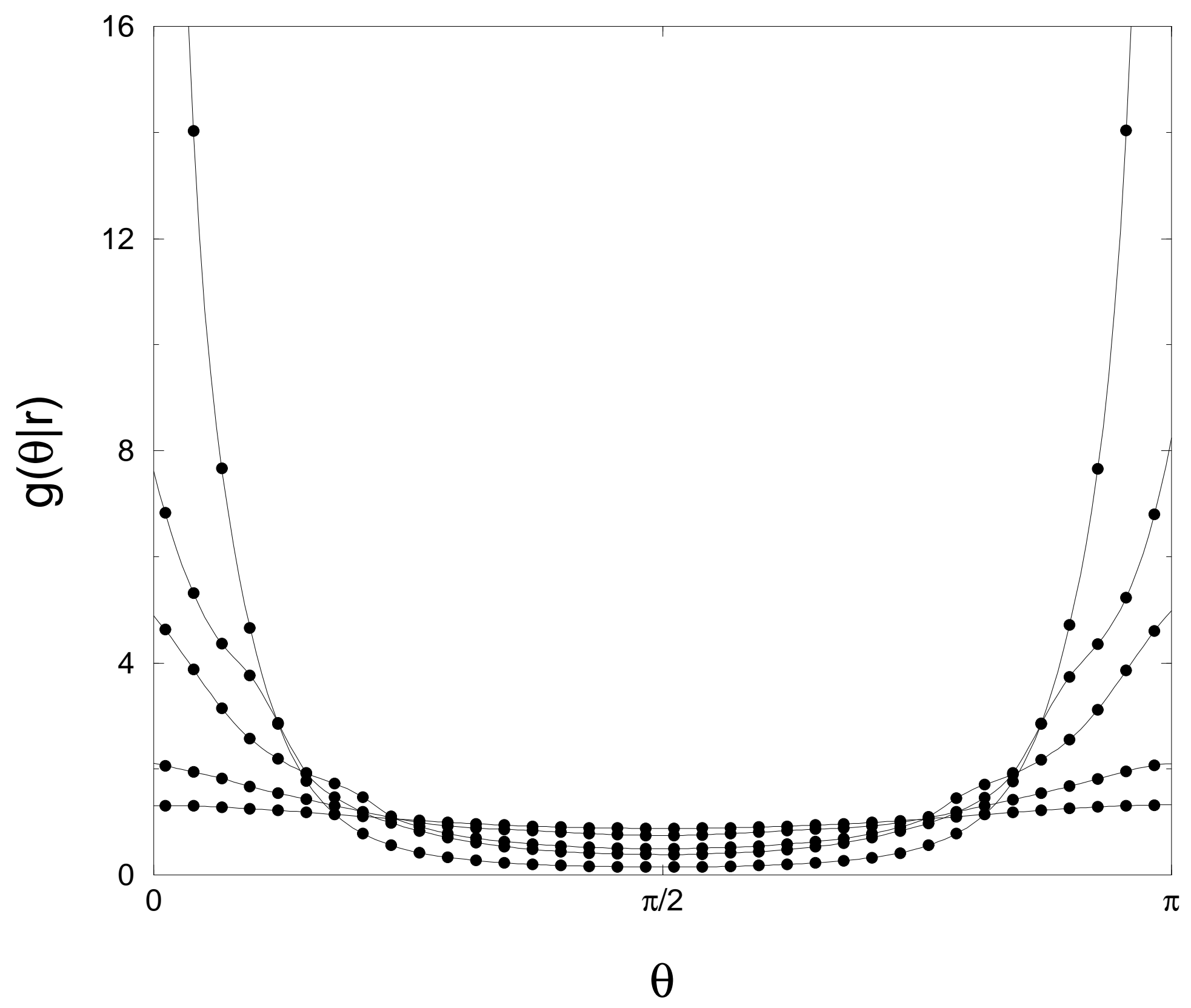




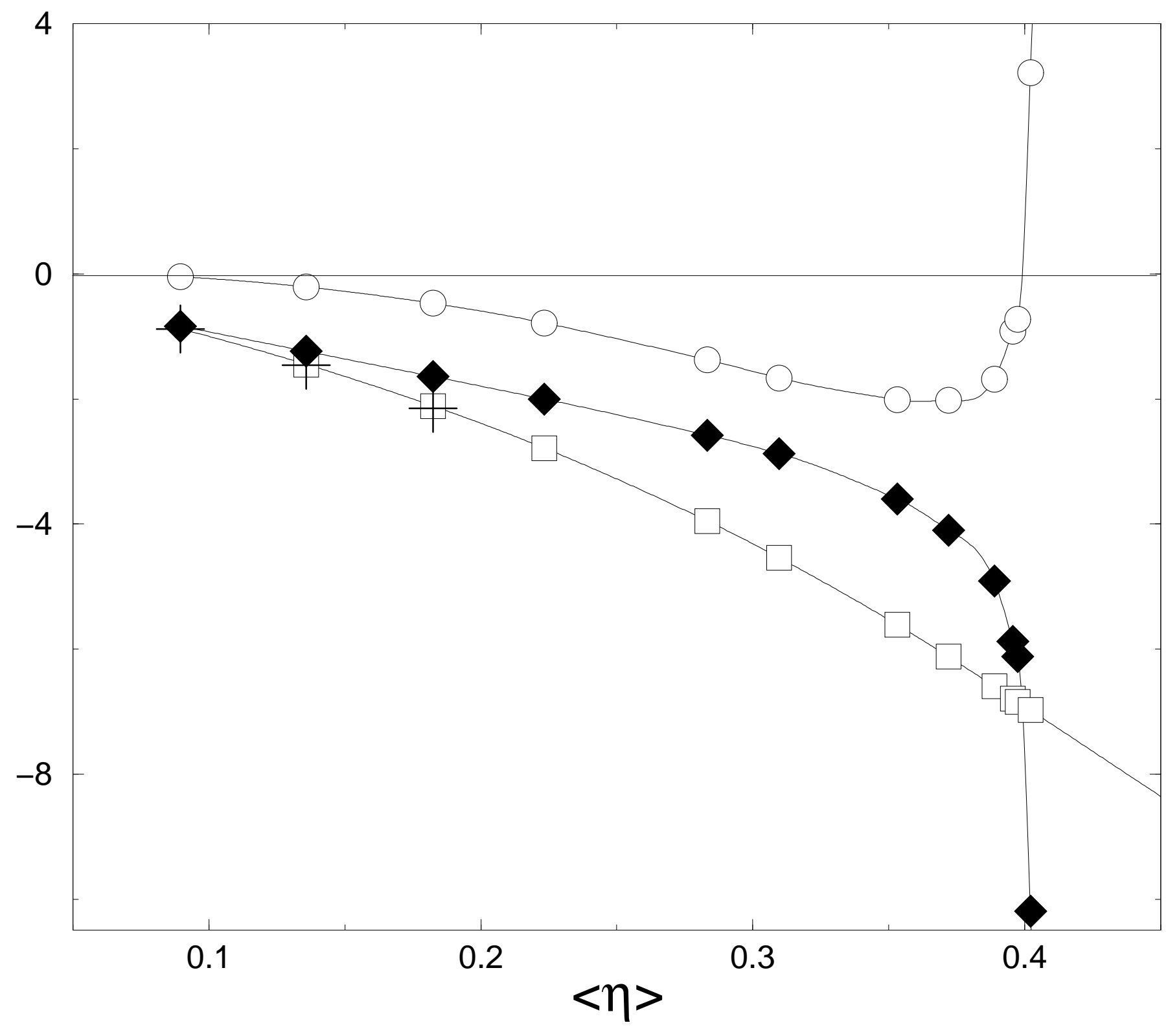




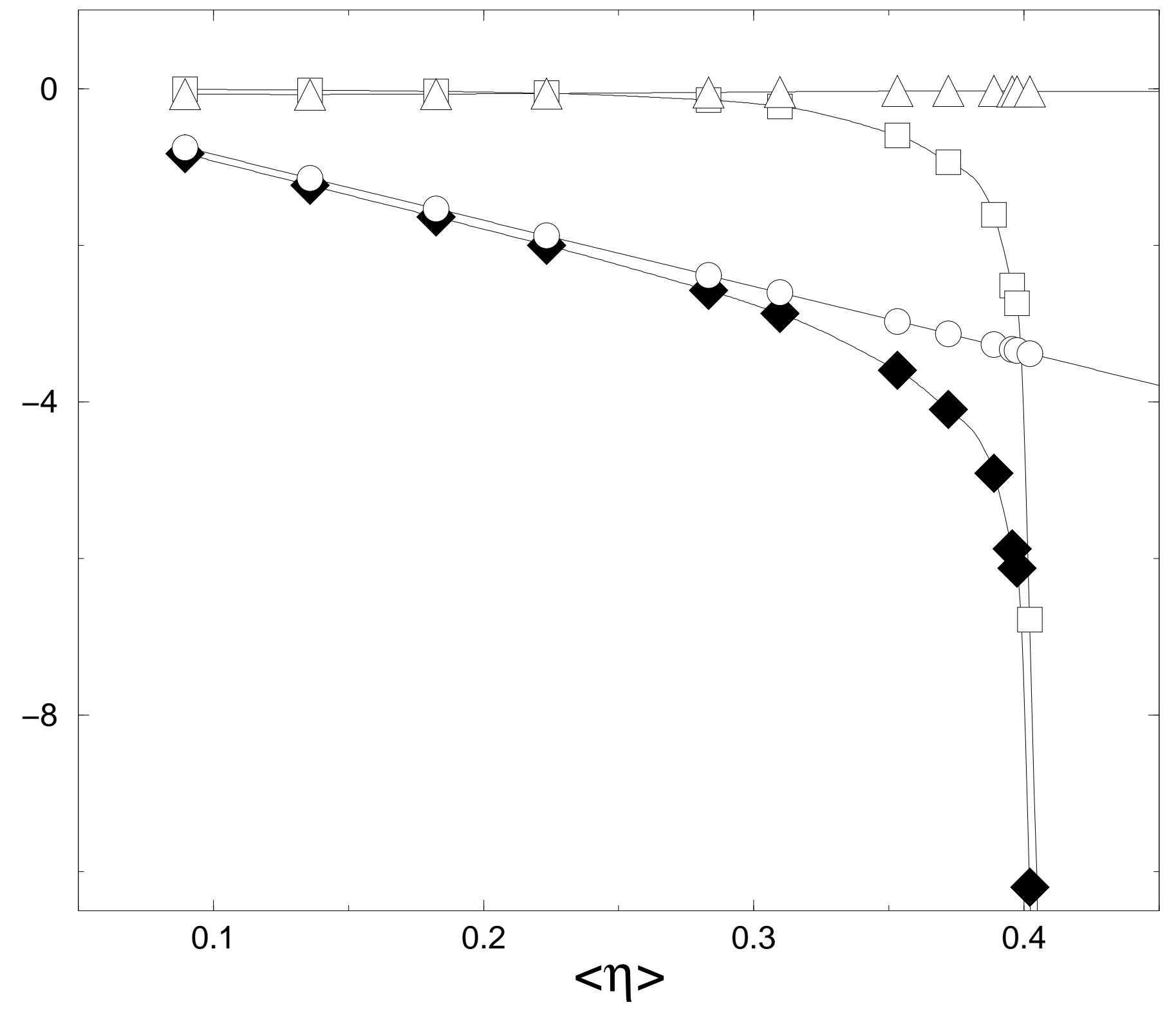




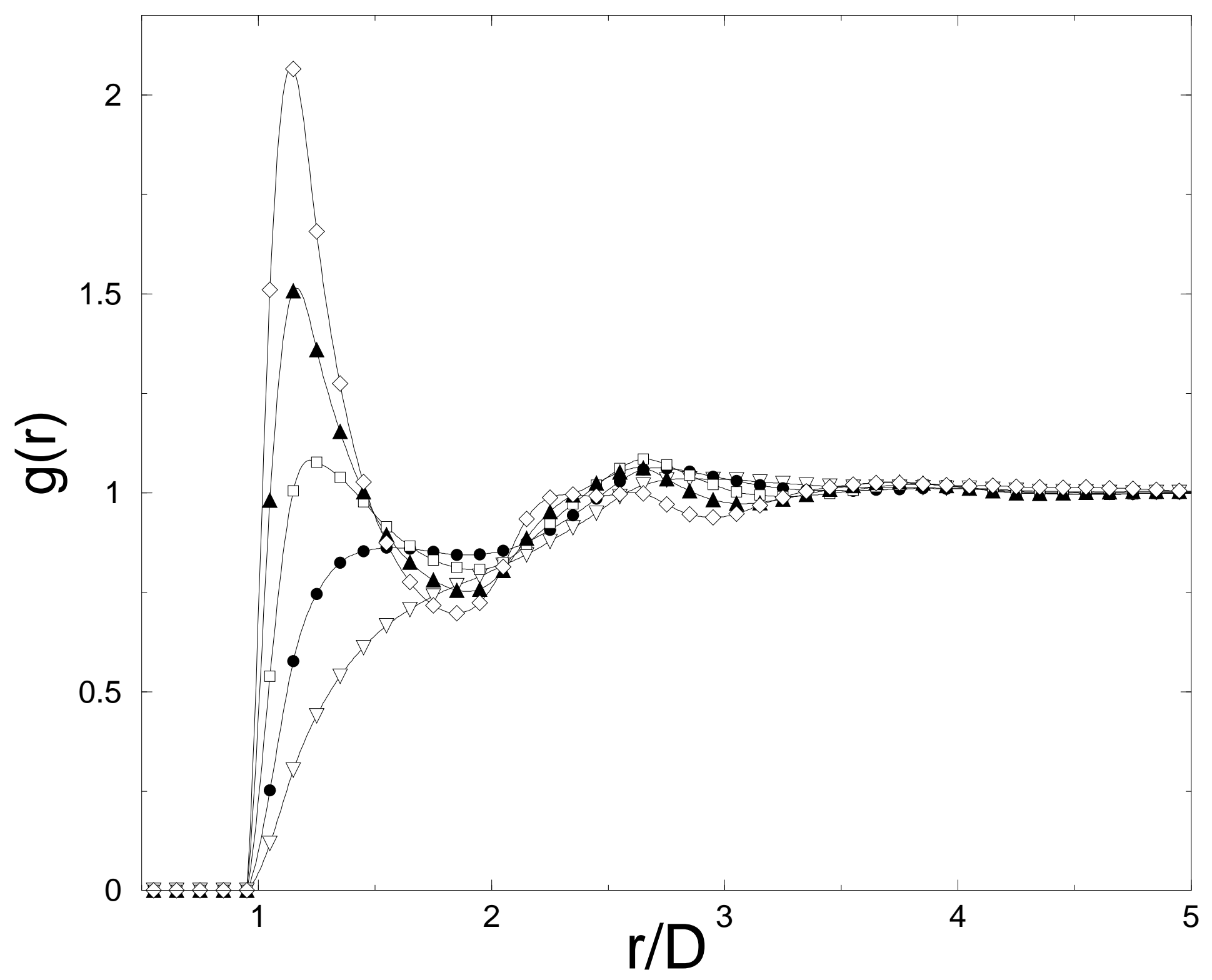




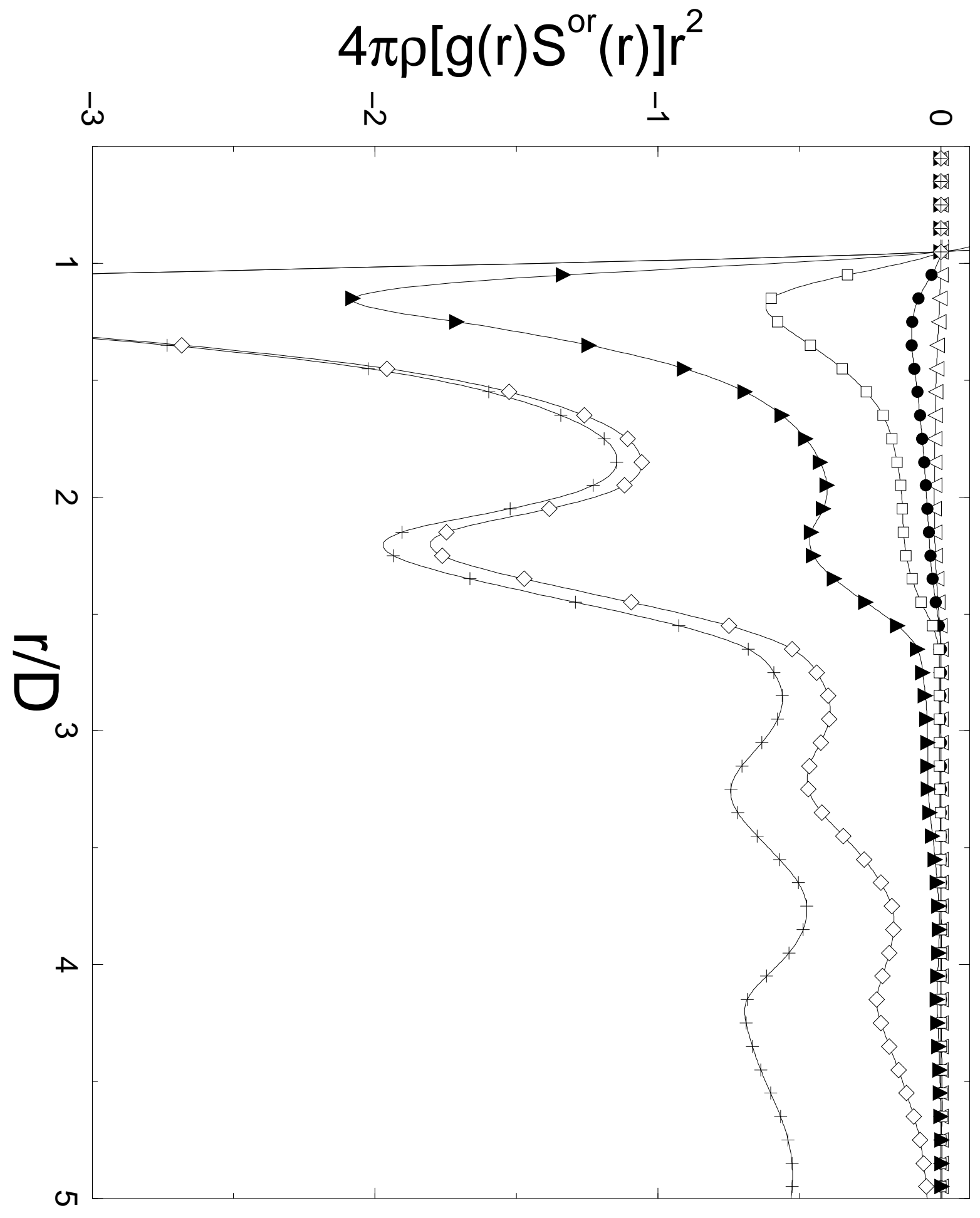




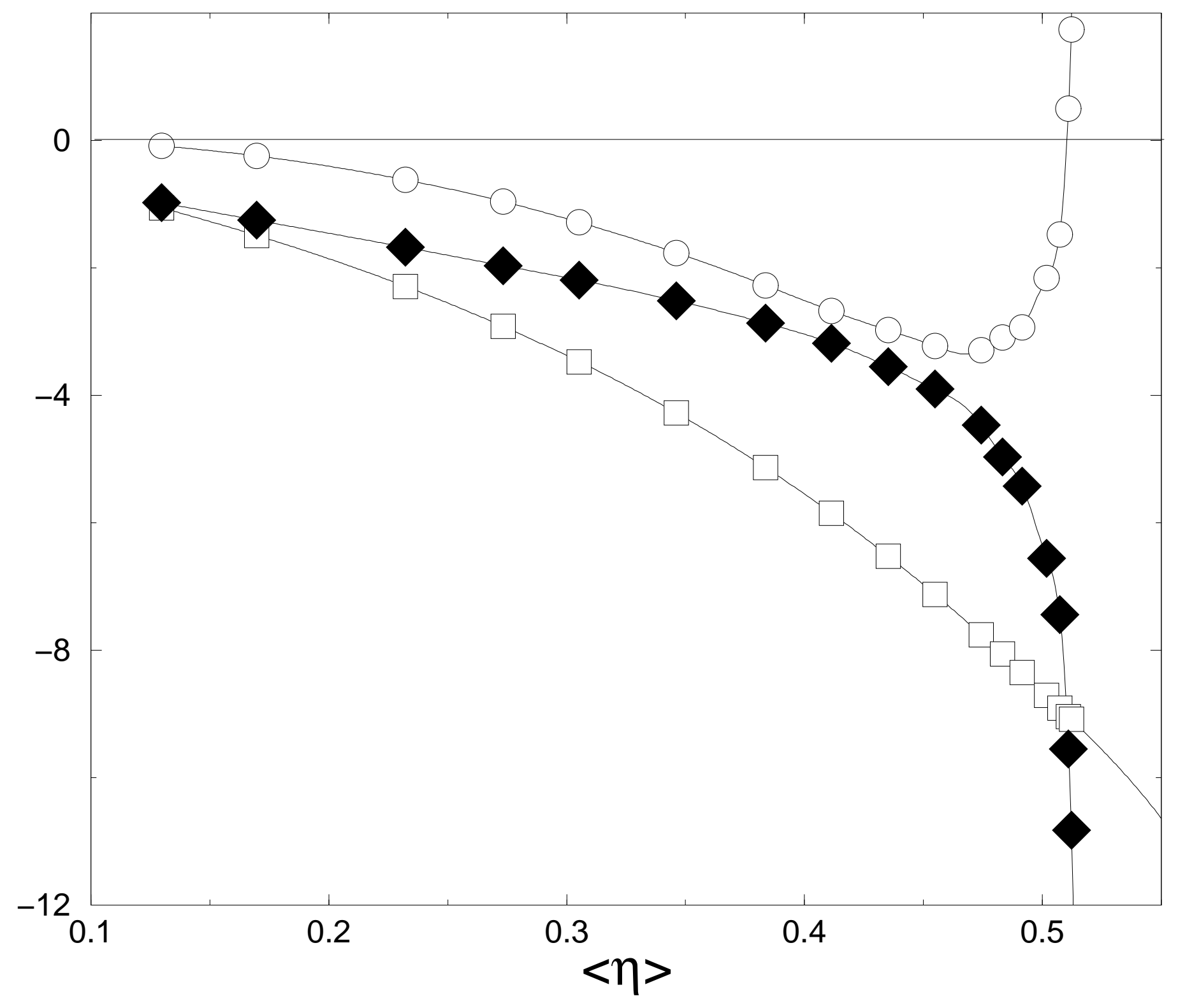




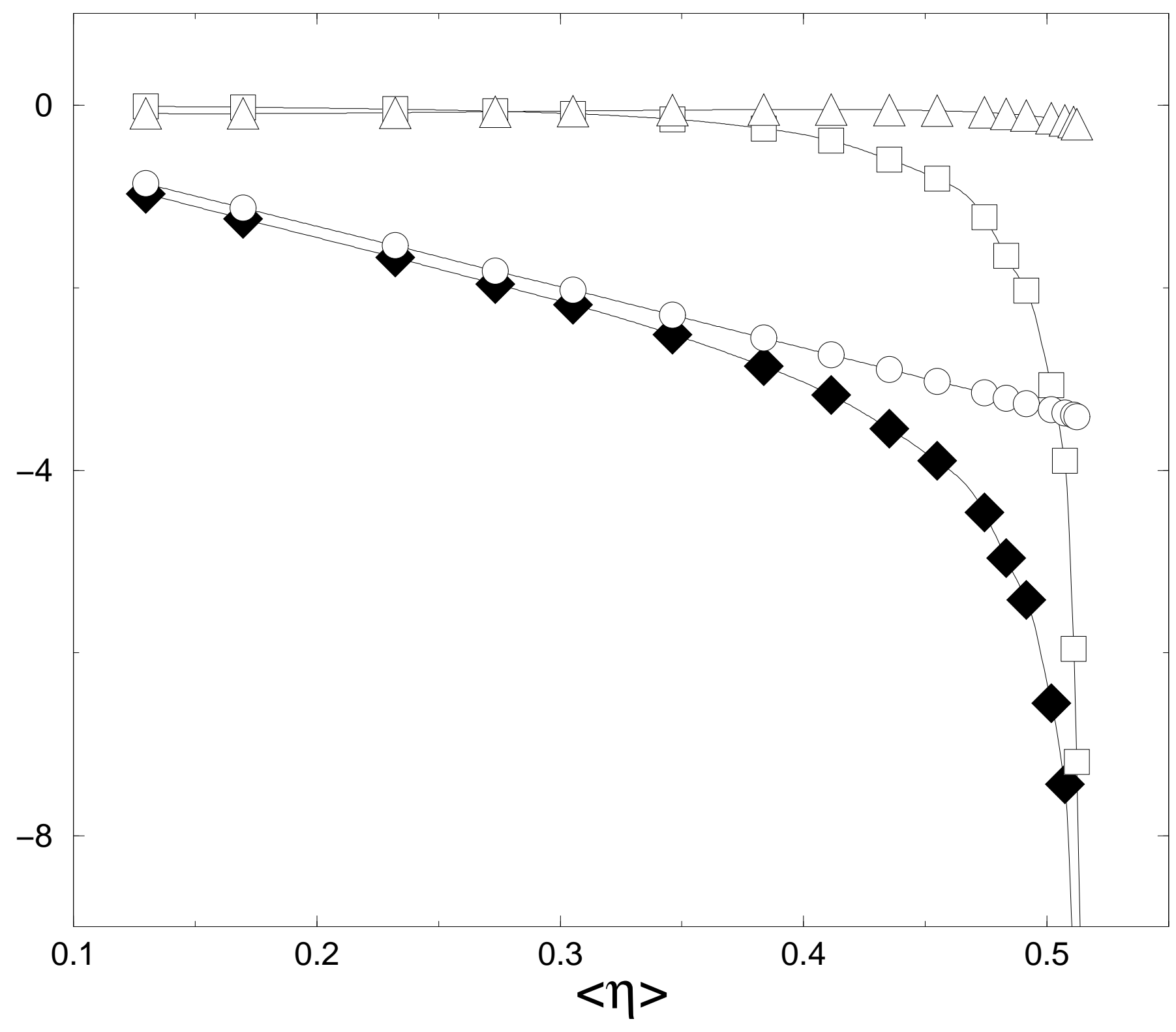




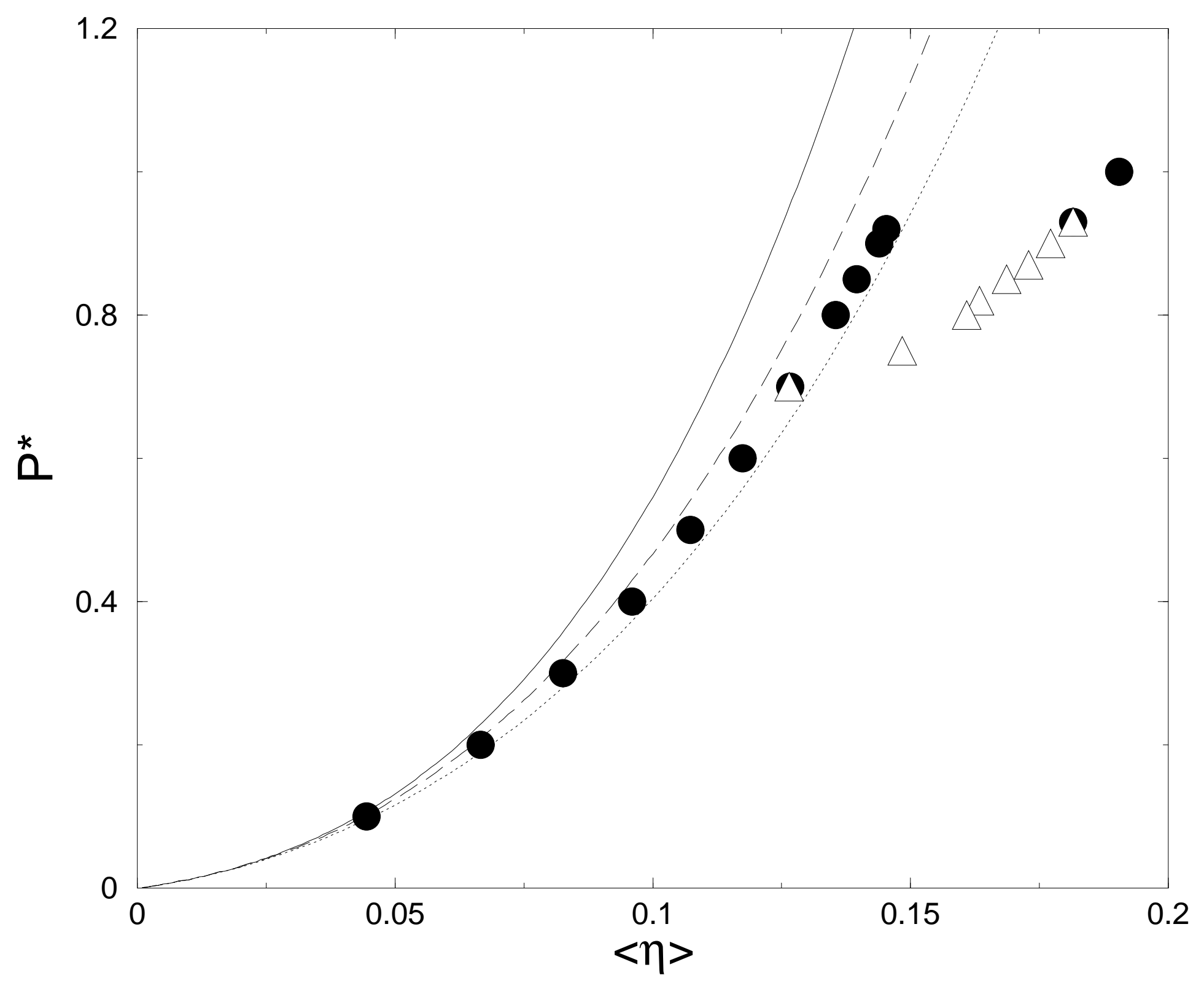




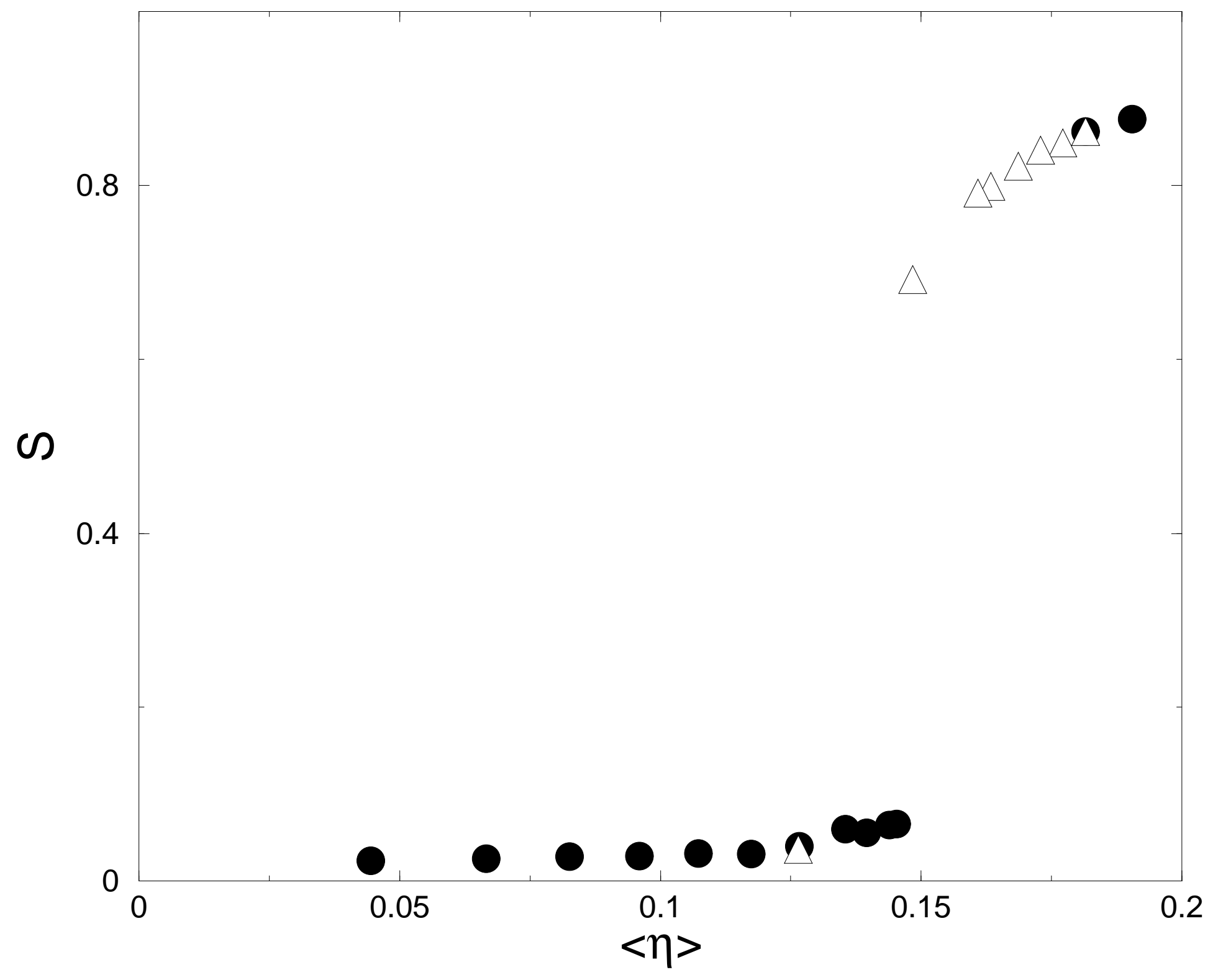




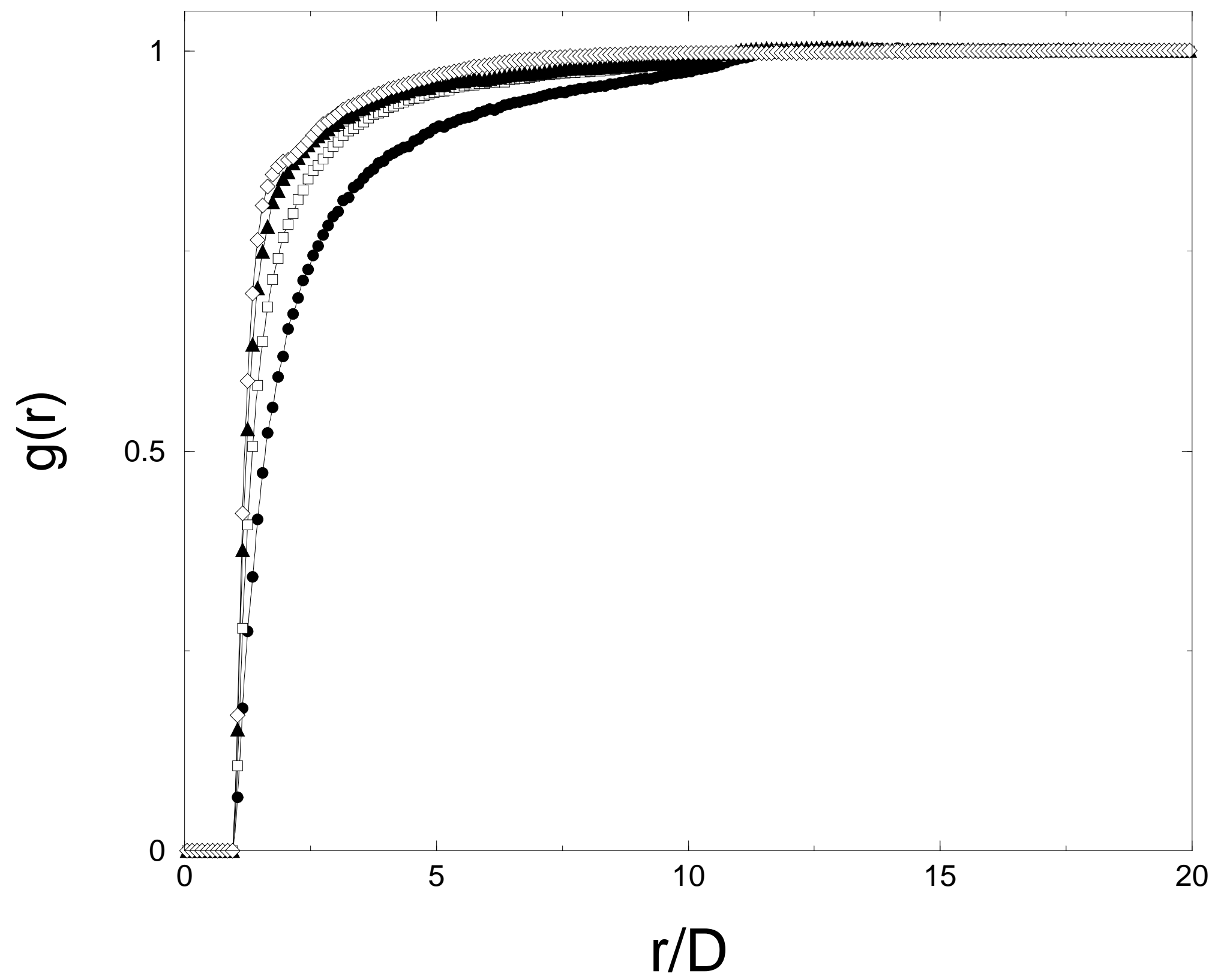




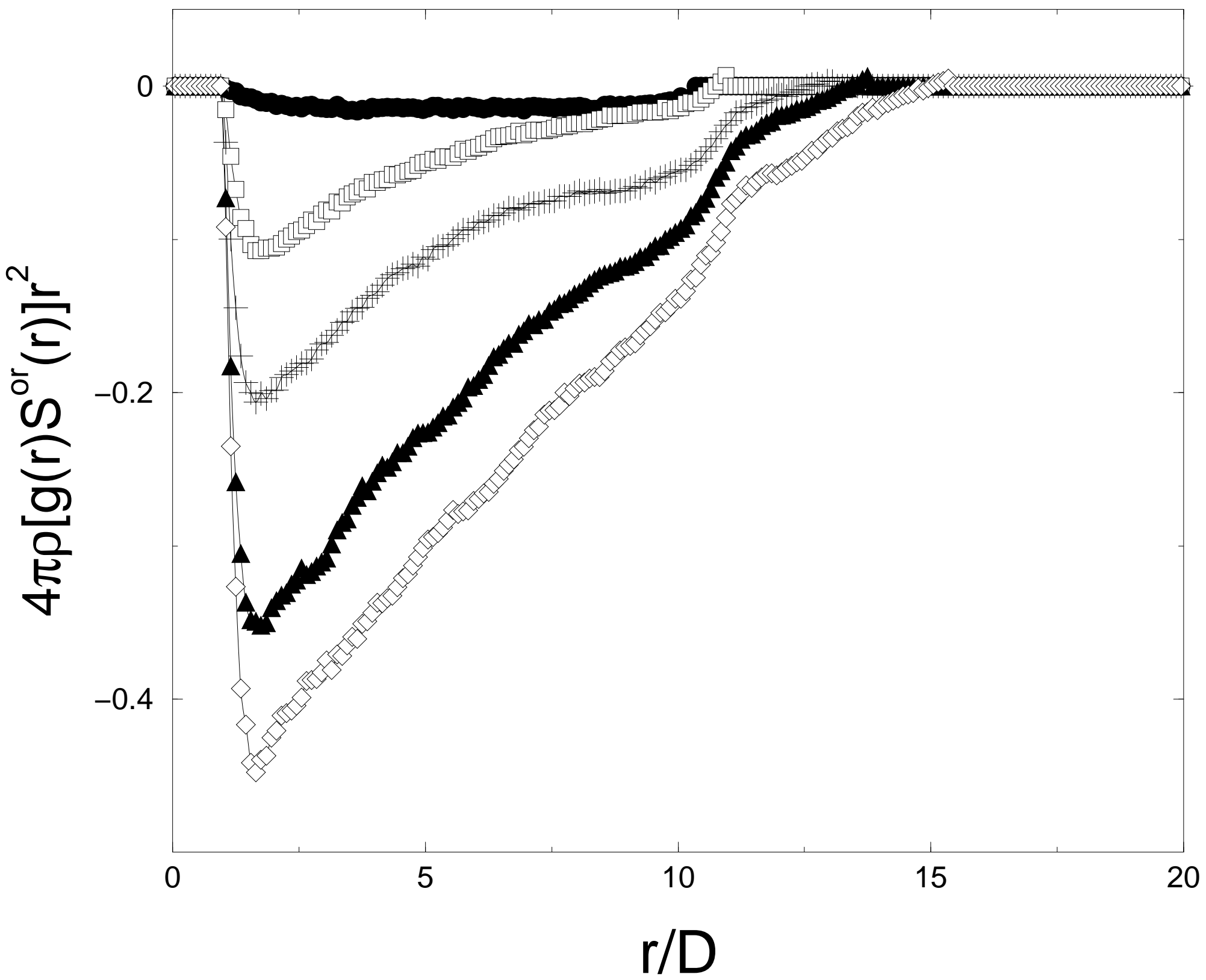




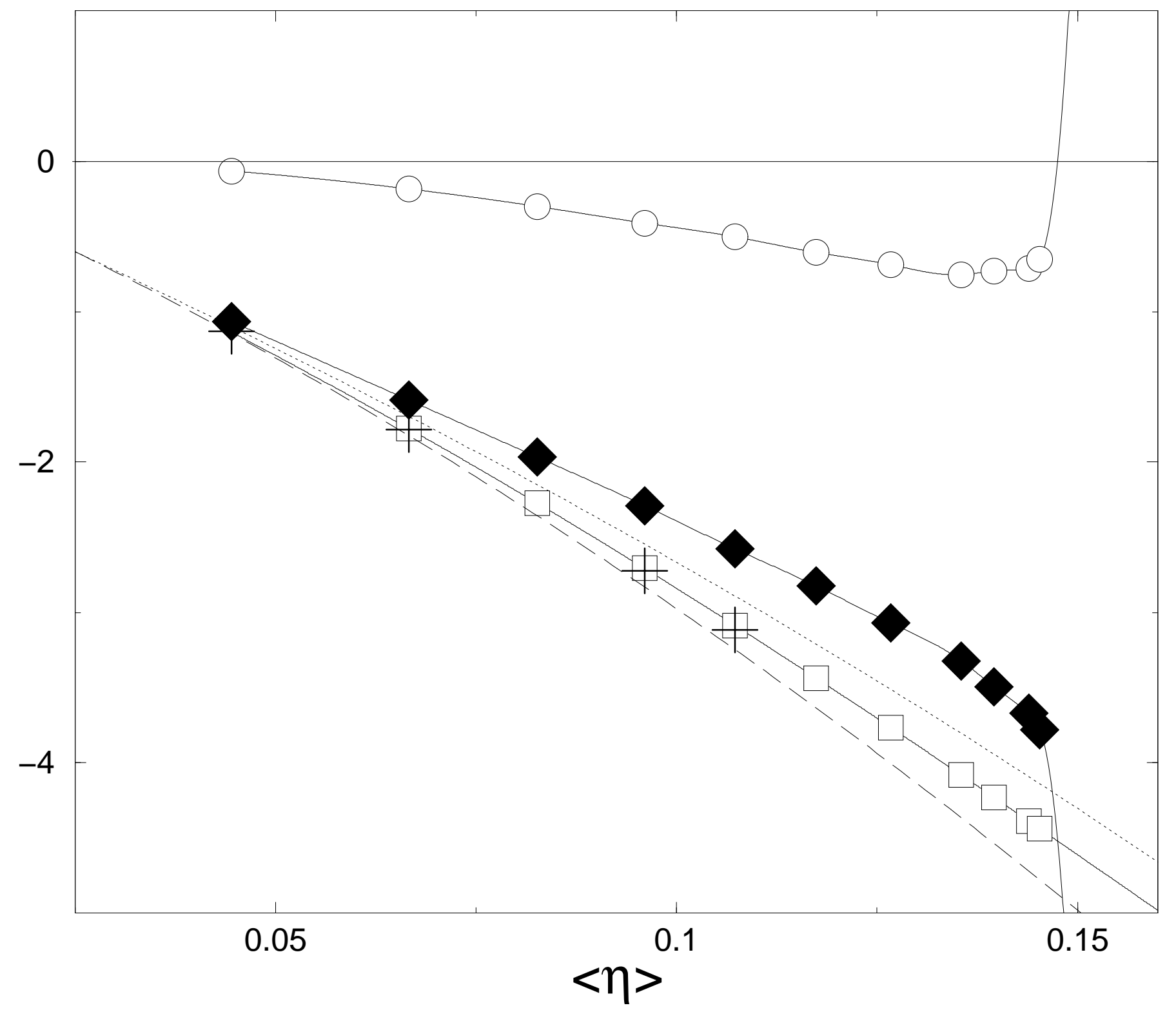




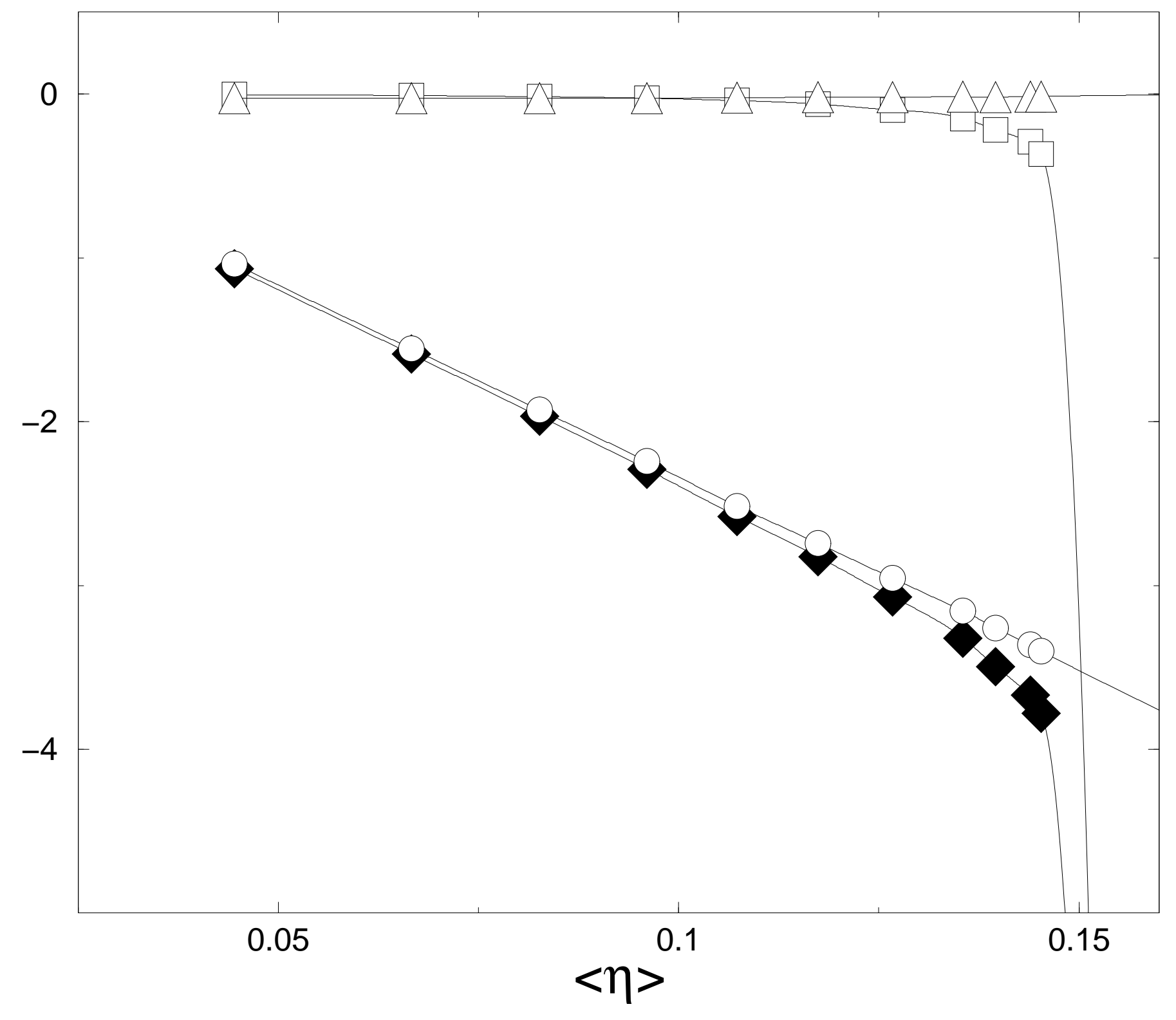




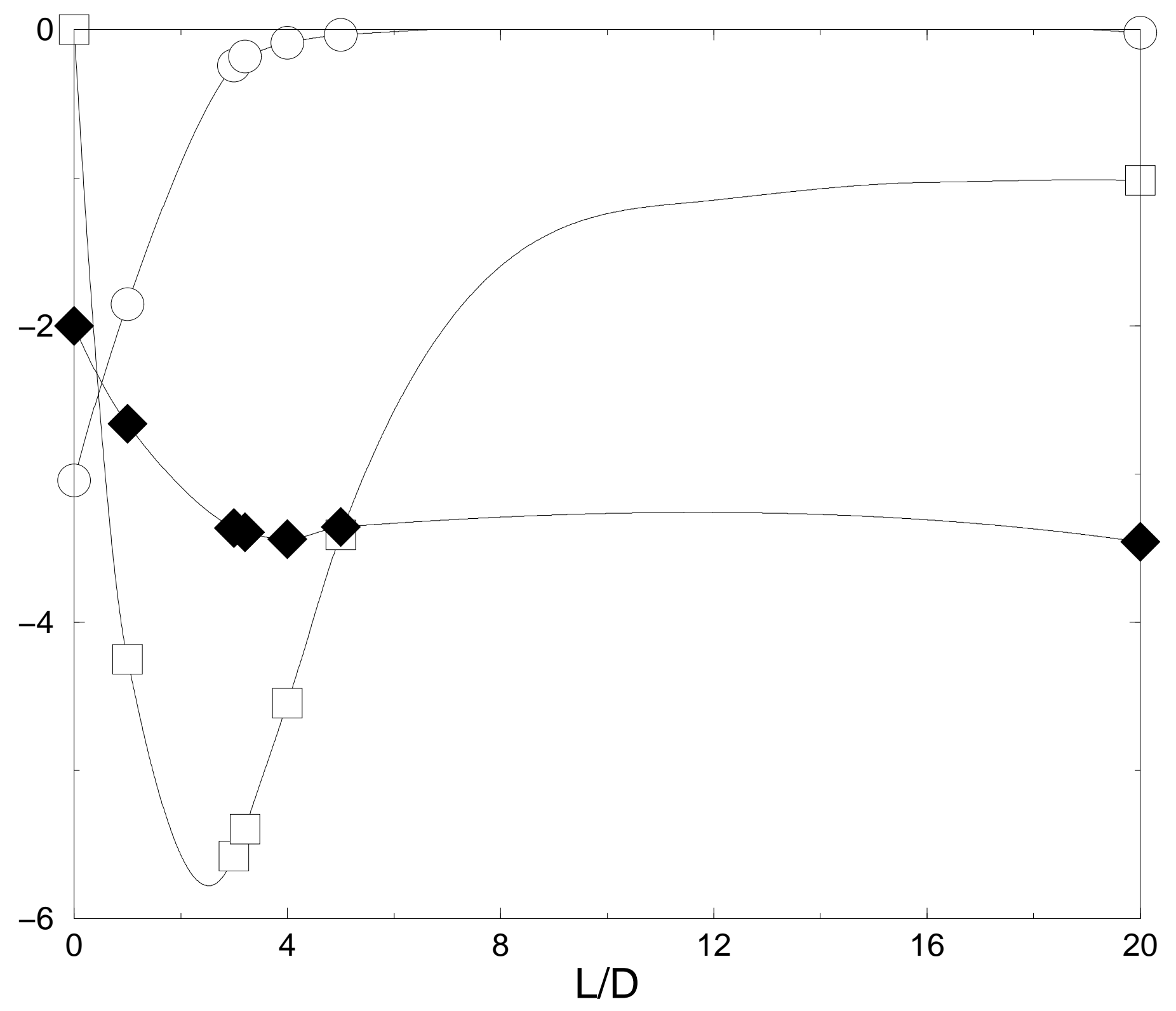

